\title{
A cut finite element method for a model of pressure in fractured media
}

\author{
Erik Burman ${ }^{1}$. Peter Hansbo ${ }^{2}$. Mats G. Larson ${ }^{3}$ \\ Received: 2 June 2020 / Revised: 16 October 2020 / Accepted: 16 October 2020 / \\ Published online: 31 October 2020 \\ (c) The Author(s) 2020
}

\begin{abstract}
We develop a robust cut finite element method for a model of diffusion in fractured media consisting of a bulk domain with embedded cracks. The crack has its own pressure field and can cut through the bulk mesh in a very general fashion. Starting from a common background bulk mesh, that covers the domain, finite element spaces are constructed for the interface and bulk subdomains leading to efficient computations of the coupling terms. The crack pressure field also uses the bulk mesh for its representation. The interface conditions are a generalized form of conditions of Robin type previously considered in the literature which allows the modeling of a range of flow regimes across the fracture. The method is robust in the following way: (1) Stability of the formulation in the full range of parameter choices; and (2) Not sensitive to the location of the interface in the background mesh. We derive an optimal order a priori error estimate and present illustrating numerical examples.
\end{abstract}

Mathematics Subject Classification $65 \mathrm{~N} 30 \cdot 65 \mathrm{~N} 12 \cdot 65 \mathrm{~N} 15$

\section{Introduction}

The numerical modelling of flow in fractured porous media is important both in environmental science and in industrial applications. It is therefore not surprising that it is currently receiving increasing attention from the scientific computing community.

\footnotetext{
$凶 \quad$ Peter Hansbo peter.hansbo@ju.se

Erik Burman

e.burman@ucl.ac.uk

Mats G. Larson

mats.larson@umu.se

1 Department of Mathematics, University College London, Gower Street, London WC1E 6BT, UK

2 Department of Mechanical Engineering, Jönköping University, 55111 Jönköping, Sweden

3 Department of Mathematics and Mathematical Statistics, Umeå University, 90187 Umeå, Sweden
} 
Here we are interested in models where the fractures are modelled as embedded surfaces of dimension $d-1$ in a $d$ dimensional bulk domain. Models on this type of geometries of mixed dimension are typically obtained by averaging the flow equations across the width of the fracture and introducing suitable coupling conditions for the modelling of the interaction with the bulk flow. Such reduced models have been derived for instance in $[1,25,29]$. The coupling conditions in these models typically take the form of a Robin type condition. The physical properties of the coupling enters as parameters in this interface condition. The size of these parameters can vary with several orders of magnitude depending on the physical properties of the crack and of the material in the porous matrix. This makes it challenging to derive methods that both are flexible with respect to mesh geometries and robust with respect to coupling conditions. A wide variety of different strategies for the discretisation of fractured porous media flow has been proposed in the literature. One approach is to use a method that allows for nonconforming coupling between the bulk mesh and the fracture mesh [3], or even arbitrary polyhedral elements in the bulk mesh in order to be able to mesh the fractures easily. This latter approach has been developed using discontinuous Galerkin methods [2], virtual element methods [18] and high order hybridised methods [14].

Herein we will consider an unfitted approach, drawing on previous work $[4,11$, 13] where flow in fractured porous media was modelled in the situation where the pressure is a globally continuous function. When using unfitted finite element methods, the bulk mesh can be created completely independently of the fractures. Instead the finite element space is modified locally to allow for discontinuities across fractures and interface conditions are typically imposed weakly, or using methods similar to Nitsche's method. For other recent work using unfitted methods we refer to [27], where a stabilized Lagrange multiplier method is considered for the interface coupling and [15] where a mixed method is considered for the Darcy's equations both in the bulk and on the surface.

The upshot here, compared to [11] is that the pressure in the crack has its own approximation field, allowing accurate approximation of problems where there is a pressure jump between the bulk and the fracture, and that the interface conditions are imposed in a way allowing for the full range of parameter values in the Robin condition, without loss of stability or order of approximation. We use the variant of the interface modelling considered in [29], that was also recently applied for the numerical modelling in [2]. In these models we may obtain a wide range of parameter values in the interface condition and we therefore develop a method which handle the full range of values and produces approximations with optimal order convergence. The approach is inspired by the work of Stenberg [26] and may be viewed as a version of the Nitsche method that can handle Robin type conditions and which converges to the standard Nitsche method when the Robin parameter tends to infinity. Previous applications of this approach in the context of fitted finite elements include [22,37].

The finite element spaces are constructed starting from a standard mesh equipped with a finite element space. For each geometric domain (subdomains and interface) we mark all the elements intersected by the domain and then we restrict the finite element space to that set to form a finite element space for each domain. This procedure leads to cut finite elements and we use stabilization to ensure that the resulting form associated with the method is coercive and that the stiffness matrix is well conditioned. The 
stabilization is of face or ghost penalty type $[6,7,28]$, and is added both to the bulk and interface spaces. Previous related work work on cut finite element methods include the interface problem [21]; overlapping meshes [23]; coupled bulk-surface problems [8, 11,12,20]; mixed dimensional problems [9], and surface partial differential equations [7,32]. For a general introduction to cut finite element methods we refer to [4].

The outline of the paper is as follows: In Sect. 2 we introduce the model problem, show an elliptic regularity result which is robust with respect to the critical parameters in the interface condition, and discuss the relation between our formulation of the interface conditions and previous work; in Sect. 3 we formulate the cut finite element method; in Sect. 4 we prove the basic properties of the formulation and in particular an optimal order a priori error estimate which is uniform in the full range of interface parameters; and in Sect. 5 we present numerical results.

\section{The model problem}

\subsection{Governing equations}

Let $\Omega$ be a convex polygonal domain in $\mathbb{R}^{d}, d=2$ or 3 , with boundary $\partial \Omega$ and exterior unit normal $n$. Let $\Gamma$ be a smooth embedded interface in $\Omega$ and let $n_{\Gamma}$ be a unit normal to $\Gamma$, which partitions $\Omega$ into two subdomains $\Omega_{1}$ and $\Omega_{2}$ with exterior unit normals $n_{1}$ and $n_{2}$. We assume that $\Gamma$ is a closed surface without boundary residing in the interior of $\Omega$, more precisely we assume that there is $\delta_{0}>0$ such that the distance between $\Gamma$ and $\partial \Omega$ is larger than $\delta$. We consider for simplicity the case with homogeneous Dirichlet conditions on $\partial \Omega$.

The problem takes the form: find $u_{i}: \Omega_{i} \rightarrow \mathbb{R}$ and $u_{\Gamma}: \Gamma \rightarrow \mathbb{R}$ such that

$$
\begin{aligned}
-\nabla \cdot A_{i} \nabla u_{i} & =f_{i} & \text { in } \Omega_{i} & \\
-\nabla_{\Gamma} \cdot A_{\Gamma} \nabla_{\Gamma} u_{\Gamma} & =f_{\Gamma}-\llbracket n \cdot A \nabla u \rrbracket & & \text { on } \Gamma \\
n \cdot A \nabla u+B\left(u-u_{\Gamma}\right) & =0 & & \text { on } \Gamma \\
u & =0 & & \text { on } \partial \Omega
\end{aligned}
$$

Here the jump (or sum) of the normal fluxes is defined by

$$
\llbracket n \cdot A \nabla v \rrbracket=\sum_{i=1}^{2} n_{i} \cdot A_{i} \nabla v_{i}
$$

In the interface condition (3), $B$ is a $2 \times 2$ symmetric matrix valued function with eigenvalues $\lambda_{i}$ such that $\lambda_{i} \in\left[c_{\lambda}, \infty\right)$, with $c_{\lambda}$ a positive constant, which means that $B$ is uniformly positive definite on $\Gamma$,

$$
c_{\lambda}\|x\|_{\mathbb{R}^{2}}^{2} \leq x \cdot B \cdot x \quad \forall x \in \mathbb{R}^{2}
$$


We also used the notation

$$
n \cdot A \nabla v=\left[\begin{array}{l}
n_{1} \cdot A_{1} \nabla v_{1} \\
n_{2} \cdot A_{2} \nabla v_{2}
\end{array}\right], \quad v-v_{\Gamma}=\left[\begin{array}{l}
v_{1}-v_{\Gamma} \\
v_{2}-v_{\Gamma}
\end{array}\right]
$$

and thus in component form (3) reads

$$
\left[\begin{array}{l}
n_{1} \cdot A_{1} \nabla u_{1} \\
n_{2} \cdot A_{2} \nabla u_{2}
\end{array}\right]+B\left[\begin{array}{l}
u_{1}-u_{\Gamma} \\
u_{2}-u_{\Gamma}
\end{array}\right]=\left[\begin{array}{l}
0 \\
0
\end{array}\right]
$$

The coefficients $A_{1}, A_{2}$, are smooth uniformly positive definite symmetric $d \times d$ matrices, $A_{\Gamma}$ is smooth tangential to $\Gamma$ and uniformly positive definite on the tangent space of $\Gamma$, so that

$$
\sum_{i=1}^{2}\left\|\nabla v_{i}\right\|_{\Omega_{i}}^{2}+\left\|\nabla_{\Gamma} v_{\Gamma}\right\|_{\Gamma}^{2} \lesssim \sum_{i=1}^{2}\left(A_{i} \nabla v_{i}, \nabla v_{i}\right)_{\Omega_{i}}+\left(A_{\Gamma} \nabla_{\Gamma} v_{\Gamma}, \nabla_{\Gamma} v_{\Gamma}\right)_{\Gamma}
$$

where $\lesssim$ denotes less or equal up to a constant. Finally, we assume $f_{i} \in L_{2}\left(\Omega_{i}\right)$ and $f_{\Gamma} \in L_{2}(\Gamma)$.

Remark 1 Several generalizations are possible on the external boundary. For instance, we may let the interface intersect the boundary of $\Omega$. In this case we let $v$ denote the unit exterior conormal to $\Gamma \cap \partial \Omega$, i.e. $v$ is tangent to $\Gamma$ and normal to $\partial \Omega \cap \Gamma$, and we assume that $v \cdot n \geq c>0$ for some constant $c$ so that the interface is transversal to $\partial \Omega$. We may then enforce the Dirichlet condition $u_{\Gamma}=g_{\Gamma}$ on $\partial \Omega \cap \Gamma$ (see [10]) or some other standard boundary condition.

Remark 2 In practical modeling we may want to take the thickness of the interface inte account. Assuming that the permeability matrix in an interface of thickness $t$ takes the form

$$
\left.A\right|_{U_{t / 2}(\Gamma)}=A_{\Gamma}^{e}+a_{\Gamma}^{e} n_{\Gamma} \otimes n_{\Gamma}
$$

where $n_{\Gamma}$ is a unit normal vector field to $\Gamma, U_{t / 2}(\Gamma)$ is the set of points with distance less than $t / 2$ to $\Gamma, v^{e}$ denotes the extension of a function $v$ on $\Gamma$ that is constant in the normal direction, $A_{\Gamma}$ is the tangential tangential permeability tensor, and finally $a_{\Gamma, n}$ is the permeability across the interface. Also assuming that $f=f_{\Gamma}^{e}$ and $u=u^{e}$ in $U_{t / 2}(\Gamma)$, the equation on the interface (2) may be modelled as follows

$$
-\nabla_{\Gamma} \cdot t A_{\Gamma} \nabla_{\Gamma} u_{\Gamma}=t f_{\Gamma}-\llbracket n \cdot A \nabla u \rrbracket \quad \text { on } \Gamma
$$

Note that the last term on the right hand side does not scale with $t$ since it accounts for flow into the crack from the bulk domains.

Remark 3 We comment on how our interface condition (3) relates to the condition in [29] and later reformulated, see [2], in terms of averages and jumps of the bulk fields 
across the interface. The interface conditions in [29], Eqs. (3.18) and (3.19), take the form

$$
\begin{aligned}
& \xi n_{1} \cdot A_{1} \nabla v_{1}-(1-\xi) n_{2} \cdot A_{2} \nabla v_{2}=\alpha\left(v_{\Gamma}-v_{1}\right) \\
& \xi n_{2} \cdot A_{2} \nabla v_{2}-(1-\xi) n_{1} \cdot A_{1} \nabla v_{1}=\alpha\left(v_{\Gamma}-v_{2}\right)
\end{aligned}
$$

where $\xi$ and $\alpha$ are parameters. The parameter $\alpha$ is related to physical properties of the interface as follows

$$
\alpha=\frac{2 a_{\Gamma, n}}{t}
$$

where $a_{\Gamma, n}$ is the permeability coefficient across the interface $\Gamma$ and $t$ is the thickness of the interface, see (3.8) in [29]. In matrix form we obtain

$$
\left[\begin{array}{cc}
\xi & \xi-1 \\
\xi-1 & \xi
\end{array}\right]\left[\begin{array}{l}
n_{1} \cdot A_{1} \nabla v_{1} \\
n_{2} \cdot A_{2} \nabla v_{2}
\end{array}\right]+\left[\begin{array}{cc}
\alpha & 0 \\
0 & \alpha
\end{array}\right]\left[\begin{array}{l}
v_{1}-v_{\Gamma} \\
v_{2}-v_{\Gamma}
\end{array}\right]=0
$$

which leads to

$$
B=\frac{1}{2 \xi-1}\left[\begin{array}{cc}
\xi & 1-\xi \\
1-\xi & \xi
\end{array}\right]\left[\begin{array}{ll}
\alpha & 0 \\
0 & \alpha
\end{array}\right]=\frac{\alpha}{2 \xi-1}\left[\begin{array}{cc}
\xi & 1-\xi \\
1-\xi & \xi
\end{array}\right]
$$

We note that we have the eigen pairs

$$
B e_{1}=\underbrace{\frac{\alpha}{2 \xi-1}}_{\lambda_{1}} e_{1}, \quad B e_{2}=\underbrace{\alpha}_{\lambda_{2}} e_{2}
$$

with the corresponding eigen vectors defined by

$$
e_{1}=\frac{1}{\sqrt{2}}\left[\begin{array}{l}
1 \\
1
\end{array}\right] \quad \text { and } \quad e_{2}=\frac{1}{\sqrt{2}}\left[\begin{array}{c}
1 \\
-1
\end{array}\right]
$$

and thus $B$ is positive definite for $\xi>1 / 2$, singular for $\xi=1 / 2$, and indefinite for $\xi<1 / 2$. It is therefore natural to consider the case when $\alpha>0$ and $\xi>1 / 2$. We remark that when $\alpha$ tends to infinity (zero) both eigenvalues tend to infinity (zero) and when $\xi$ tends to $1 / 2$ from above one eigenvalue tends to infinity. It is therefore important to construct a method which is robust in the full range $\lambda_{i} \in(0, \infty)$ of possible values for the two eigenvalues.

To see the relation to the formulation of the interface conditions in [2] we first note that we have the expansions

$$
\begin{aligned}
& {\left[\begin{array}{l}
n_{1} \cdot A_{1} \nabla v_{1} \\
n_{2} \cdot A_{2} \nabla v_{2}
\end{array}\right]=2^{-1 / 2} \llbracket n \cdot A \nabla \rrbracket e_{1}+2^{1 / 2}\langle n \cdot A \nabla v\rangle e_{2}} \\
& {\left[\begin{array}{l}
v_{1}-v_{\Gamma} \\
v_{2}-v_{\Gamma}
\end{array}\right]=2^{1 / 2}\left(\langle v\rangle-v_{\Gamma}\right) e_{1}+2^{-1 / 2} \llbracket v \rrbracket e_{2}}
\end{aligned}
$$


where the jumps and averages of the bulk fields across the the interface are defined by

$$
\begin{gathered}
\llbracket n \cdot A \nabla v \rrbracket=\sum_{i=1}^{2} n_{i} \cdot A_{i} \nabla v_{i}, \quad \llbracket v \rrbracket=v_{1}-v_{2} \\
\langle n \cdot A \nabla v\rangle=\frac{1}{2}\left(n_{1} \cdot A_{1} \nabla v_{1}-n_{2} \cdot A_{2} \nabla v_{2}\right), \quad\left\langle v 》=\frac{1}{2}\left(v_{1}+v_{2}\right)\right.
\end{gathered}
$$

Using the expansions (19) and (20) together with (17) and matching the coefficients associated with each eigenvector we obtain the interface conditions

$$
\begin{gathered}
\llbracket n \cdot A \nabla v \rrbracket+\frac{2 \alpha}{2 \xi-1}\left(\langle v\rangle-v_{\Gamma}\right)=0 \\
\langle n \cdot A \nabla v\rangle+\frac{\alpha}{2} \llbracket v \rrbracket=0
\end{gathered}
$$

which are precisely the conditions used in [2].

Remark 4 The geometry of the interface may be generalized in several ways, which is needed in practical modeling of systems of cracks. For instance, we may consider bifurcating cracks where a so called Kirchhoff condition holds along the intersection, cracks that meet the boundary, and cracks which are piecewise smooth. We refer to $[5,8,11,24]$, for details on how to construct CutFEM for bifurcating cracks and how to handle the Kirchhoff condition weakly in a systematic manner. The regularity of the exact solution may be locally lower due to nonconvex corners and edges and therefore there may be a need for adaptive mesh refinement. More difficult to handle are cracks with boundary in the interior of the domain since they may lead to singularities in the bulk field, see [16,17], and furthermore the geometry of the crack tip plays an important since it determines the boundary conditions at the crack tip see [30] for details. The properties of the solutions to problems with crack boundaries are interesting future research topics. We do however remark that the finite element method may be directly extended to cracks with boundaries if we assume a homogeneous Neumann condition at the crack tip, see [31] for numerical studies using a simpler but related method.

\subsection{Weak form}

Define the function spaces

$$
\begin{aligned}
V & =V_{1} \oplus V_{2} \oplus V_{\Gamma} \\
V_{i} & =\left\{v_{i} \in H^{1}\left(\Omega_{i}\right): v=0 \text { on } \partial \Omega \cap \partial \Omega_{i}\right\} \quad i=1,2 \\
V_{\Gamma} & =H^{1}(\Gamma)
\end{aligned}
$$

and let $v \in V$ denote the vector $v=\left(v_{1}, v_{2}, v_{\Gamma}\right)$. We will also use the notation $\tilde{V}$ for functions $v \in V$ such that $v_{i} \in H^{\frac{3}{2}+\epsilon}\left(\Omega_{i}\right), i=1,2$, and $v_{\Gamma} \in H^{\frac{3}{2}+\epsilon}(\Gamma)$, with 
$\epsilon>0$. Using partial integration on $\Omega_{i}$ we obtain

$$
\begin{aligned}
\sum_{i=1}^{2} & \left(f_{i}, v_{i}\right)_{\Omega_{i}} \\
= & \sum_{i=1}^{2}\left(-\nabla \cdot A_{i} \nabla u_{i}, v_{i}\right)_{\Omega_{i}} \\
= & \sum_{i=1}^{2}\left(A_{i} \nabla u_{i}, \nabla v_{i}\right)_{\Omega_{i}}-\left(n_{i} \cdot A_{i} \nabla u_{i}, v_{i}\right)_{\partial \Omega_{i}} \\
= & \sum_{i=1}^{2}\left(A_{i} \nabla u_{i}, \nabla v_{i}\right)_{\Omega_{i}} \\
& -\left(n_{i} \cdot A_{i} \nabla u_{i}, v_{i}-v_{\Gamma}\right)_{\partial} \Omega_{i} \cap \Gamma-\left(n_{i} \cdot A_{i} \nabla u_{i}, v_{\Gamma}\right)_{\partial} \Omega_{i} \cap \Gamma \\
= & \sum_{i=1}^{2}\left(A_{i} \nabla u_{i}, \nabla v_{i}\right)_{\Omega_{i}}-\left(n \cdot A \nabla u, v-v_{\Gamma}\right)_{\Gamma}-\left(\llbracket n \cdot A \nabla u \rrbracket, v_{\Gamma}\right)_{\Gamma} \\
= & \sum_{i=1}^{2}\left(A_{i} \nabla u_{i}, \nabla v_{i}\right)_{\Omega_{i}}+\left(B\left(u-u_{\Gamma}\right), v-v_{\Gamma}\right)_{\Gamma} \\
& +\left(A_{\Gamma} \nabla u_{\Gamma}, \nabla v_{\Gamma}\right)_{\Gamma}-\left(f_{\Gamma}, v_{\Gamma}\right)_{\Gamma}
\end{aligned}
$$

Thus we arrive at the weak problem: find $u=\left(u_{1}, u_{2}, u_{\Gamma}\right) \in V$ such that

$$
\mathcal{A}(u, v)=L(v) \quad \forall v \in V
$$

where the forms are defined by

$$
\begin{aligned}
\mathcal{A}(u, v)= & \sum_{i=1}^{2}\left(A_{i} \nabla u_{i}, \nabla v_{i}\right)_{\Omega_{i}} \\
& +\left(A_{\Gamma} \nabla_{\Gamma} u_{\Gamma}, \nabla_{\Gamma} v_{\Gamma}\right)_{\Gamma}+\left(B\left(u-u_{\Gamma}\right), v-v_{\Gamma}\right)_{\Gamma} \\
L(v)= & \sum_{i=1}^{2}\left(f_{i}, v_{i}\right)_{\Omega_{i}}+\left(f_{\Gamma}, v_{\Gamma}\right)_{\Gamma}
\end{aligned}
$$

\subsection{Existence, uniqueness, and regularity}

Introducing the energy norm

$$
\|v\|^{2}=\sum_{i=1}^{2}\|v\|_{H^{1}\left(\Omega_{i}\right)}^{2}+\left\|v_{\Gamma}\right\|_{H^{1}(\Gamma)}^{2}+\left\|v-v_{\Gamma}\right\|_{B, \Gamma}^{2}
$$


on $V$, we directly find using a Poincaré inequality and the Cauchy-Schwarz inequality that the form $A$ is coercive and continuous

$$
\|v\|^{2} \lesssim \mathcal{A}(v, v), \quad \mathcal{A}(v, w) \lesssim\|v\|\|\| w \|
$$

Furthermore, $L$ is a continuous functional on $V$ and it follows from the Lax-Milgram Lemma that there is a unique solution in $V$ to (36).

Lemma 1 In the case considered here where $\Gamma$ is a smooth, closed interface the model problem (36) satisfies the elliptic regularity estimate

$$
\left\|u_{1}\right\|_{H^{2}\left(\Omega_{1}\right)}+\left\|u_{2}\right\|_{H^{2}\left(\Omega_{2}\right)}+\left\|u_{\Gamma}\right\|_{H^{2}(\Gamma)} \lesssim\left\|f_{1}\right\|_{\Omega_{1}}+\left\|f_{2}\right\|_{\Omega_{2}}+\left\|f_{\Gamma}\right\|_{\Gamma}
$$

Under the additional assumption that $B$ is a constant matrix and $A_{i}=a_{i} \mathbb{I}_{[d \times d]}$ $i=1,2$ and $A_{\Gamma}=a_{\Gamma} \mathbb{I}_{[(d-1) \times(d-1)]}$ with $a_{i} \in \mathbb{R}^{+}$and $\mathbb{I}_{[d \times d]}$ the identity matrix, then the constant in (42) is independent of the coefficients of $B$.

Remark 5 The assumptions that $A_{i}=a_{i} \mathbb{I}_{[d \times d]}$ and $B$ is constant along $\Gamma$ can be relaxed to smoothly varying coefficients, with additional technical work. See "Appendix C" for the case of variable $B$ with uniformly bounded derivatives. We have not included the full analysis of the general case in the manuscript to keep the presentation at a reasonable level of complexity.

To prove (42) we first recall a partial integration formula from [19], see Eq. 3.1.1.1 on p. 134. For completeness we include a proof based on tangential calculus, which is in line with the notation used in this paper, in "Appendix A".

Lemma 2 Let $\omega \subset \mathbb{R}^{d}$ be a domain with $C^{2}$ boundary $\partial \omega$ and exterior unit normal $n$. For $w \in\left[H^{2}(\omega)\right]^{d}$ it holds

$$
\begin{aligned}
(\nabla \cdot w, \nabla \cdot w)_{\omega}= & (w \otimes \nabla, \nabla \otimes w)_{\omega}+2\left(w_{n}, \operatorname{div}_{T} w_{T}\right)_{\partial \omega} \\
& +\left(w_{T}, w_{T}\right)_{\kappa, \partial \omega}+\left(w_{n}, w_{n}\right)_{\operatorname{tr}(\kappa), \partial \omega}
\end{aligned}
$$

Here $w=w_{T}+w_{n} n$ is the decomposition of $w$ into the tangential and normal components of the vector field $w$ in an open neighborhood of the boundary; $\kappa=$ $\nabla \otimes n=\nabla^{2} \zeta$ is the tangential curvature tensor of $\partial \omega$ and $\zeta$ is the signed distance function of $\partial \omega$ such that $n=\nabla \zeta$; and div $v_{T}(w)=\operatorname{tr}\left(w \otimes \nabla_{T}\right)$ is the surface divergence on $\partial \omega$.

Proof of Lemma 1 The proof consists of three steps: (1) Use the Lax-Milgram lemma to show existence and $B$ independent stability in $H^{1}$. (2) Show that the $H^{1}$ solution is in fact in $H^{2}$. (3) Apply the partial integration identity (43) to derive a $B$ independent estimate for the $H^{2}$ norm.

We will neglect the exterior boundary and focus our attention on the interface condition. The extension to the convex polygonal exterior boundary can be handled using standard techniques, see [19, Theorem 4.3.1.4] for a proof in the two dimensional case. For brevity we will also employ the notation $\nabla_{n} v=n \cdot \nabla v$ in the proof. 
Step 1. Using the Lax-Milgram lemma there is a unique solution $\left(u_{1}, u_{2}, u_{\Gamma}\right) \in$ $H^{1}\left(\Omega_{2}\right) \oplus H^{1}\left(\Omega_{1}\right) \oplus H^{1}(\Gamma)$, which satisfies the energy bound

$$
\sum_{i=1}^{2}\left\|u_{i}\right\|_{H^{1}\left(\Omega_{i}\right)}^{2}+\left\|u_{\Gamma}\right\|_{H^{1}(\Gamma)}^{2}+\left\|u-u_{\Gamma}\right\|_{B, \Gamma}^{2} \lesssim \sum_{i=1}^{2}\left\|f_{i}\right\|_{\Omega_{i}}^{2}+\left\|f_{\Gamma}\right\|_{\Gamma}^{2}
$$

with hidden constant independent of $B$.

Step 2. Since $u_{i} \in H^{1}\left(\Omega_{i}\right), i=1,2$, and $u_{\Gamma} \in H^{1}(\Gamma)$ we have $\left.B\left(u-u_{\Gamma}\right)\right|_{\Gamma} \in$ $\left[H^{\frac{1}{2}}(\Gamma)\right]^{2}$ and using (3), $\nabla_{n} u \in\left[H^{\frac{1}{2}}(\Gamma)\right]^{2}$. This means that the right hand side of (2) is in $L^{2}$ and hence $u_{\Gamma} \in H^{2}(\Gamma)$ by elliptic regularity. Considering once again (3) we see that in each subdomain the solution coincides with a single domain solution with a Robin condition with data in $H^{\frac{1}{2}}(\Gamma)$ on $\Gamma$. By the elliptic regularity of the Robin problem we can then conclude that $u_{i} \in H^{2}\left(\Omega_{i}\right)$ and therefore $\left(u_{1}, u_{2}, u_{\Gamma}\right) \in$ $H^{2}\left(\Omega_{1}\right) \otimes H^{2}\left(\Omega_{2}\right) \otimes H^{2}(\Gamma)$.

Step 3. Setting $\omega=\Omega_{i}$ and $w_{i}=a_{i}^{\frac{1}{2}} \nabla u_{i}$ in the partial integration identity (43) and summing the contributions from $\Omega_{1}$ and $\Omega_{2}$ we obtain

$$
\begin{aligned}
\sum_{i=1}^{2} a_{i}\left\|\Delta u_{i}\right\|_{\Omega_{i}}^{2}= & \sum_{i=1}^{2} a_{i}\left\|\nabla^{2} u_{i}\right\|_{\Omega_{i}}^{2}+2 \sum_{i=1}^{2}\left(a_{i} \nabla_{n} u_{i}, \Delta_{\Gamma} u_{i}\right)_{\partial \Omega_{i} \cap \Gamma} \\
& +\sum_{i=1}^{2}\left(a_{i} \nabla_{\Gamma} u_{i}, \nabla_{\Gamma} u_{i}\right)_{\kappa, \Gamma}+\sum_{i=1}^{2}\left(a_{i} \nabla_{n} u_{i}, \nabla_{n} u_{i}\right)_{\operatorname{tr}(\kappa), \Gamma}
\end{aligned}
$$

Recalling that $a_{i} \Delta u_{i}=f_{i}$ and rearranging the terms we

$$
\begin{aligned}
& \sum_{i=1}^{2} a_{i}\left\|\nabla^{2} u_{i}\right\|_{\Omega_{i}}^{2}+2 \underbrace{\sum_{i=1}^{2}\left(a_{i} \nabla_{n} u_{i}, \Delta_{\Gamma} u_{i}\right)_{\partial \Omega_{i} \cap \Gamma}}_{I} \\
& \leq \sum_{i=1}^{2} a_{i}^{-1}\left\|f_{i}\right\|_{\Omega_{i}}^{2}+\sum_{i=1}^{2} \underbrace{\left|\left(a_{i} \nabla_{\Gamma} u_{i}, \nabla_{\Gamma} u_{i}\right)_{\kappa, \Gamma}\right|}_{I I_{i}}+\sum_{i=1}^{2} \underbrace{\left|\left(a_{i} \nabla_{n} u_{i}, \nabla_{n} u_{i}\right)_{\operatorname{tr}(\kappa), \Gamma}\right|}_{I I I_{i}}
\end{aligned}
$$

We now proceed with estimates from below of term $I$, and estimates of $I I_{i}$ and $I I I_{i}$. We let $C$ denote a generic constant non necessarily the same in all occurrences.

Term I. Adding and subtracting $u_{\Gamma}$ we get

$$
\begin{aligned}
I & =\sum_{i=1}^{2}\left(a_{i} \nabla_{n_{i}} u_{i}, \Delta_{\Gamma}\left(u_{i}-u_{\Gamma}\right)\right)_{\partial \Omega_{i}}+\sum_{i=1}^{2}\left(a_{i} \nabla_{n_{i}} u_{i}, \Delta_{\Gamma} u_{\Gamma}\right)_{\partial} \Omega_{i} \\
& =-\left(B\left[u-u_{\Gamma}\right], \Delta_{\Gamma}\left[u-u_{\Gamma}\right]\right)_{\Gamma}+\sum_{i=1}^{2}\left(\llbracket a \nabla_{n} u \rrbracket, \Delta_{\Gamma} u_{\Gamma}\right)_{\Gamma}
\end{aligned}
$$




$$
\begin{aligned}
& =\left(\nabla_{\Gamma} B\left[u-u_{\Gamma}\right], \nabla_{\Gamma}\left[u-u_{\Gamma}\right]\right)_{\Gamma}+\sum_{i=1}^{2}\left(a_{\Gamma} \Delta_{\Gamma} u_{\Gamma}+f_{\Gamma}, \Delta_{\Gamma} u_{\Gamma}\right)_{\Gamma} \\
& \geq\left\|\nabla_{\Gamma}\left[u-u_{\Gamma}\right]\right\|_{B, \Gamma}^{2}+a_{\Gamma} \frac{1}{2}\left\|\Delta_{\Gamma} u_{\Gamma}\right\|_{\Gamma}^{2}-\frac{1}{2} a_{\Gamma}^{-1}\left\|f_{\Gamma}\right\|_{\Gamma}^{2}
\end{aligned}
$$

Here we used the assumption that $B$ is constant, which gives

$$
\begin{aligned}
-\left(B\left[u-u_{\Gamma}\right], \Delta_{\Gamma}\left[u-u_{\Gamma}\right]\right)_{\Gamma} & =\left(\nabla_{\Gamma}\left(B\left[u-u_{\Gamma}\right]\right), \nabla_{\Gamma}\left[u-u_{\Gamma}\right]\right)_{\Gamma} \\
& =\left(B \nabla_{\Gamma}\left[u-u_{\Gamma}\right], \nabla_{\Gamma}\left[u-u_{\Gamma}\right]\right)_{\Gamma} \\
& =\left\|\nabla_{\Gamma}\left[u-u_{\Gamma}\right]\right\|_{B, \Gamma}^{2}
\end{aligned}
$$

For the second term

$$
\begin{aligned}
\left(a_{\Gamma} \Delta_{\Gamma} u_{\Gamma}+f_{\Gamma}, \Delta_{\Gamma} u_{\Gamma}\right)_{\Gamma} \geq & a_{\Gamma}\left\|\Delta_{\Gamma} u_{\Gamma}\right\|_{\Gamma}^{2}-\left\|f_{\Gamma}\right\|_{\Gamma}\left\|\Delta_{\Gamma} u_{\Gamma}\right\|_{\Gamma} \\
& \geq \frac{1}{2} a_{\Gamma}\left\|\Delta_{\Gamma} u_{\Gamma}\right\|_{\Gamma}^{2}-\frac{1}{2} a_{\Gamma}^{-1}\left\|f_{\Gamma}\right\|_{\Gamma}^{2}
\end{aligned}
$$

Term $I_{i}$. Using interpolation [35, Proposition 3.1] followed by the trace inequality

$$
\|w\|_{H^{s}(\Gamma)} \leq C\|w\|_{H^{s+1 / 2}\left(\Omega_{i}\right)}, \quad s>0
$$

we get

$$
\begin{aligned}
I I_{i} & =\left|\left(a_{i} \nabla_{\Gamma} u_{i}, \nabla_{\Gamma} u_{i}\right)_{\kappa, \Gamma}\right| \\
& \leq\|\kappa\|_{L^{\infty}(\Gamma)}\left(a_{i} \nabla_{\Gamma} u_{i}, \nabla_{\Gamma} u_{i}\right)_{\Gamma} \\
& \leq C a_{i}\left\|u_{i}\right\|_{H^{3 / 2}(\Gamma)}\left\|u_{i}\right\|_{H^{1 / 2}(\Gamma)} \\
& \leq C a_{i}\left\|u_{i}\right\|_{H^{2}\left(\Omega_{i}\right)}\left\|u_{i}\right\|_{H^{1}\left(\Omega_{i}\right)} \\
& \leq \frac{1}{4} a_{i}\left\|u_{i}\right\|_{H^{2}\left(\Omega_{i}\right)}^{2}+C a_{i}\left\|u_{i}\right\|_{H^{1}\left(\Omega_{i}\right)}^{2}
\end{aligned}
$$

Term $I I I_{i}$. Again using interpolation and then by applying the trace inequalities (59) and

$$
\left\|\nabla_{n_{i}} u_{i}\right\|_{H^{-1 / 2}(\Gamma)} \leq C\left(\left\|\nabla u_{i}\right\|_{\Omega_{i}}^{2}+\left\|\Delta u_{i}\right\|_{\Omega_{i}}^{2}\right)^{1 / 2}
$$

we get

$$
\begin{aligned}
I I I_{i} & =\left|a_{i}\left(\nabla_{n} u_{i}, \nabla_{n} u_{i}\right)_{\operatorname{tr}(\kappa), \Gamma}\right| \\
& \leq\|\operatorname{tr}(\kappa)\|_{L^{\infty}(\Gamma)} a_{i}\left(\nabla_{n} u_{i}, \nabla_{n} u_{i}\right)_{\Gamma} \\
& \leq C a_{i}\left\|\nabla_{n} u_{i}\right\|_{H^{1 / 2}(\Gamma)}\left\|\nabla_{n} u_{i}\right\|_{H^{-1 / 2}(\Gamma)} \\
& \leq C a_{i}\left\|\nabla u_{i}\right\|_{H^{1}\left(\Omega_{i}\right)}\left(\left\|\nabla u_{i}\right\|_{\Omega_{i}}^{2}+\left\|\Delta u_{i}\right\|_{\Omega_{i}}^{2}\right)^{1 / 2} \\
& \leq \frac{1}{4} a_{i}\left\|u_{i}\right\|_{H^{2}\left(\Omega_{i}\right)}^{2}+C a_{i}\left(\left\|\nabla u_{i}\right\|_{\Omega_{i}}^{2}+\left\|\Delta u_{i}\right\|_{\Omega_{i}}^{2}\right)
\end{aligned}
$$

Here we used the estimate $\left\|\nabla_{n} u_{i}\right\|_{\Gamma} \leq C\left\|\nabla u_{i}\right\|_{\Omega_{i}}$, which we prove in "Appendix B". 
Conclusion. Starting from (49) and using the estimate (53) to bound term I from below and estimates (64) and (70) to bound terms $I I_{i}$ and $I I I_{i}$ from above we obtain

$$
\begin{aligned}
& \sum_{i=1}^{2} a_{i}\left\|\nabla^{2} u_{i}\right\|_{\Omega_{i}}^{2}+\left\|\nabla_{\Gamma}\left[u-u_{\Gamma}\right]\right\|_{B, \Gamma}^{2}+\frac{1}{2} a_{\Gamma}\left\|\Delta_{\Gamma} u_{\Gamma}\right\|_{\Gamma}^{2}-\frac{1}{2} a_{\Gamma}^{-1}\left\|f_{\Gamma}\right\|_{\Gamma}^{2} \\
& \leq \sum_{i=1}^{2} a_{i}^{-1}\left\|f_{i}\right\|_{\Omega_{i}}^{2}+\sum_{i=1}^{2} \frac{1}{4} a_{i}\left\|u_{i}\right\|_{H^{2}\left(\Omega_{i}\right)}^{2}+C a_{i}\left\|u_{i}\right\|_{H^{1}\left(\Omega_{i}\right)}^{2} \\
& \quad+\sum_{i=1}^{2} \frac{1}{4} a_{i}\left\|u_{i}\right\|_{H^{2}\left(\Omega_{i}\right)}^{2}+C a_{i}\left(\left\|\nabla u_{i}\right\|_{\Omega_{i}}^{2}+\left\|\Delta u_{i}\right\|_{\Omega_{i}}^{2}\right)
\end{aligned}
$$

Reorganizing the terms and using the identity $a_{i} \Delta u_{i}=f_{i}$, and writing $\left\|u_{i}\right\|_{H^{2}\left(\Omega_{i}\right)}^{2}=$ $\left\|\nabla^{2} u_{i}\right\|_{\Omega_{i}}^{2}+\left\|u_{i}\right\|_{H^{1}\left(\Omega_{i}\right)}^{2}$ we get

$$
\begin{aligned}
& \sum_{i=1}^{2} a_{i}\left\|\nabla^{2} u_{i}\right\|_{\Omega_{i}}^{2}+\left\|\nabla_{\Gamma}\left[u-u_{\Gamma}\right]\right\|_{B, \Gamma}^{2}+a_{\Gamma}\left\|\Delta_{\Gamma} u_{\Gamma}\right\|_{\Gamma}^{2} \\
& \quad \leq \frac{1}{2} a_{\Gamma}^{-1}\left\|f_{\Gamma}\right\|_{\Gamma}^{2}+\sum_{i=1}^{2} C a_{i}^{-1}\left\|f_{i}\right\|_{\Omega_{i}}^{2}+\sum_{i=1}^{2} C a_{i}\left\|u_{i}\right\|_{H^{1}\left(\Omega_{i}\right)}^{2} \\
& \quad \lesssim a_{\Gamma}^{-1}\left\|f_{\Gamma}\right\|_{\Gamma}^{2}+\sum_{i=1}^{2}\left(1+a_{i}^{-1}\right)\left\|f_{i}\right\|_{\Omega_{i}}^{2}
\end{aligned}
$$

where we used the energy stability (45) in the final step, and the hidden constant depends on the trace constants and the curvature constant $\kappa$.

\section{A robust finite element method}

\subsection{The mesh and finite element spaces}

To formulate the finite element method we introduce the following notation:

- Let $\mathcal{T}_{h, 0}$ be a quasiuniform mesh on $\Omega$ with mesh parameter $h \in\left(0, h_{0}\right]$. Define the active meshes

$$
\begin{aligned}
\mathcal{T}_{h, i} & =\left\{T \in \mathcal{T}_{h, 0}: T \cap \Omega_{i} \neq \emptyset\right\} \quad i=1,2 \\
\mathcal{T}_{h, \Gamma} & =\left\{T \in \mathcal{T}_{h, 0}: T \cap \Gamma \neq \emptyset\right\}
\end{aligned}
$$

associated with the bulk domains $\Omega_{i}, i=1,2$, and the interface $\Gamma$, and the domains covered by the meshes

$$
O_{h, i}=\cup_{T \in \mathcal{T}_{h, i}} \quad i=1,2, \quad O_{h, \Gamma}=\cup_{T \in \mathcal{T}_{h, \Gamma}}
$$


- Let

$$
\mathcal{T}_{h, i}(\Gamma)=\left\{T \in \mathcal{T}_{h, i}: T \cap \Gamma \neq \emptyset\right\}
$$

- Let $\mathcal{F}_{h, i}$ be the set of all interior faces in $\mathcal{T}_{h, i}$ associated with an element in $\mathcal{T}_{h, i}\left(\partial \Omega_{i}\right)=\left\{T \in \mathcal{T}_{h, i}: T \cap \partial \Omega_{i} \neq \emptyset\right\}$.

- Let $\mathcal{F}_{h, \Gamma}$ be the set of all interior faces in $\mathcal{T}_{h, \Gamma}$ and $\mathcal{K}_{h, \Gamma}=\{K=T \cap \Gamma: T \in$ $\left.\mathcal{T}_{h, \Gamma}\right\}$.

- Let $V_{h, 0}$ be the space of continuous piecewise linear functions on $\mathcal{T}_{h, 0}$ and define

$$
V_{h, i}=\left.V_{h, 0}\right|_{\mathcal{T}_{h, i}} \quad i=1,2, \quad V_{h, \Gamma}=\left.V_{h, 0}\right|_{\mathcal{T}_{h, \Gamma}}
$$

and

$$
V_{h}=V_{h, 1} \oplus V_{h, 2} \oplus V_{h, \Gamma}
$$

For $V_{h, 1}$ we also impose the homogeneous boundary condition on $\partial \Omega$ strongly, i.e., we require $v=0$ on $\partial \Omega$.

\subsection{Standard formulation}

The standard finite element method takes the form: find $u_{h}=\left(u_{h, 1}, u_{h, 2}, u_{h, \Gamma}\right) \in$ $V_{h}=V_{h, 1} \oplus V_{h, 2} \oplus V_{h, \Gamma}$ such that

$$
\mathcal{A}_{h}^{S}\left(u_{h}, v\right)=L(v) \quad \forall v \in V_{h}
$$

Here the form $\mathcal{A}_{h}^{S}$ is defined by

$$
\mathcal{A}_{h}^{S}=\mathcal{A}+s_{h}
$$

where $s_{h}$ is a stabilization term of the form

$$
s_{h}=s_{h, 1}+s_{h, 2}+s_{h, \Gamma}
$$

with

$$
s_{h, i}(v, w)=\sum_{F \in \mathcal{F}_{h, i}} h_{F}\left\|\zeta\left(A_{i}\right)\right\|_{\infty, F}([n \cdot \nabla v],[n \nabla w])_{F} \quad i=1,2
$$

where $\zeta(X)$ denotes the maximum eigenvalue of the matrix $X$,

$$
\begin{aligned}
s_{h, \Gamma}(v, w)= & \sum_{F \in \mathcal{F}_{h, \Gamma}} h_{F}\left\|\zeta\left(A_{\Gamma}\right)\right\|_{\infty, F \cap \Gamma}([n \cdot \nabla v],[n \cdot \nabla w])_{\mathcal{F}_{h, \Gamma}} \\
& +\sum_{T \in \mathcal{T}_{h, \Gamma}} h_{K}^{2}\left\|\zeta\left(A_{\Gamma}\right)\right\|_{\infty, K \cap \Gamma}\left(n_{\Gamma} \cdot \nabla v, n_{\Gamma} \cdot \nabla w\right)_{T \cap \Gamma}
\end{aligned}
$$

where for a face sharing two elements $T_{1}$ and $T_{2}$ the jump $[n \cdot \nabla v]$ is defined by

$$
[n \cdot \nabla v]=n_{1} \cdot \nabla v_{1}+n_{2} \cdot \nabla v_{2}
$$

where $n_{i}$ is the exterior normal to $\partial T_{i}$ and $v_{i}=\left.v\right|_{T_{i}}$. 


\subsubsection{Properties of the stabilization terms}

The rationale for the design of the stabilizing terms is that they improve the stability, while remaining consistent for sufficiently smooth solutions.

Accuracy relies on the following consistency property that is immediate from the definitions above. For any function $v \in H^{\frac{3}{2}+\epsilon}\left(O_{h, i}\right)$ there holds $s_{h, i}(v, w)=0$ for all $w \in V_{h, i}+H^{\frac{3}{2}+\epsilon}\left(O_{h, i}\right), i=1,2$. For any function $v \in H^{\frac{3}{2}+\epsilon}\left(O_{h, \Gamma}\right)$, such that $n_{\Gamma} \cdot \nabla v=0$ on $\Gamma$ there holds $s_{h, \Gamma}(v, w)=0$ for all $w \in V_{h, \Gamma}+H^{\frac{3}{2}+\epsilon}\left(O_{h, \Gamma}\right)$.

The stability properties are well known and we collect them in the following Lemma.

Lemma 3 There are constants such that

$$
\|\nabla v\|_{A_{i}, O_{h, i}}^{2} \lesssim\|\nabla v\|_{A_{i}, \Omega_{i}}^{2}+\|v\|_{S_{h, i}}^{2} i=1,2
$$

and

$$
\left\|\nabla_{\Gamma} v\right\|_{A_{\Gamma}, O_{h, \Gamma}}^{2} \lesssim\left\|\nabla_{\Gamma} v\right\|_{A_{\Gamma}, \Gamma}^{2}+\|v\|_{S_{h, \Gamma}}^{2}
$$

where we introduced the (semi) norm $\|v\|_{s_{h}}^{2}=s_{h}(v, v)$.

Proof See $[6,7,28]$, with minor modifications to account for the varying coefficients.

Remark 6 Observe that the hidden constants in Lemma 3 depend on the variation of the $A_{i}$ and $A_{\Gamma}$.

Remark 7 The finite element methods we develop in this paper directly extends to higher continuous piecewise polynomial spaces with the modification that the stabilizing terms controls jumps in higher derivatives across faces and for the surface stabilization higher order normal derivatives must be added, see [28] for full details.

\subsection{Robust formulation}

The stabilizing terms ensure robustness irrespective of the intersection of the fracture and the mesh, but they do not counter instabilities due to degenerate $B$. Our aim is to design a formulation which is robust in the case when the eigenvalues of $B$ degenerate. Indeed as we saw above as $\xi$ approaches $1 / 2, \lambda_{1}$ blows up. For clarity we recall the abstract boundary condition

$$
n \cdot A \nabla v+B\left(v-v_{\Gamma}\right)=0
$$

where we now assume that the matrix $B$ is a positive definite symmetric $2 \times 2$ matrix with eigenvalues $\lambda_{i}$ and eigenvectors $e_{i}$, such that $\lambda_{i} \in(0, \infty)$ and thus one or both eigenvalues may become very large or small. To handle this situation we instead enforce

$$
B^{-1} n \cdot A \nabla v+\left(v-v_{\Gamma}\right)=0
$$

weakly using a modified Nitsche method. This approach was originally developed in [26] where fitted finite element approximation of Robin conditions were considered. 
Derivation of an Alternative Weak Form. As before we have the identity

$$
\begin{aligned}
L(v) & =\underbrace{\sum_{i=1}^{2}\left(A_{i} \nabla u_{i}, \nabla v_{i}\right)_{\Omega_{i}}+\left(A_{\Gamma} \nabla_{\Gamma} u_{\Gamma}, \nabla_{\Gamma} v_{\Gamma}\right)_{\Gamma}}_{=: \mathcal{A}_{1}(u, v)}-\left(n \cdot A \nabla u, v-v_{\Gamma}\right)_{\Gamma} \\
& =\mathcal{A}_{1}(u, v)-\left(n \cdot A \nabla u, v-v_{\Gamma}\right)_{\Gamma}
\end{aligned}
$$

where we introduced the bilinear form $\mathcal{A}_{1}$ for brevity. To enforce the interface conditions we proceed as follows

$$
\begin{aligned}
L(v)= & \mathcal{A}_{1}(u, v)-\left(n \cdot A \nabla u, v-v_{\Gamma}\right)_{\Gamma} \\
= & \mathcal{A}_{1}(u, v)+\left(n \cdot A \nabla u, B^{-1}(n \cdot A \nabla v)\right)_{\Gamma} \\
& -\left(n \cdot A \nabla u, B^{-1}(n \cdot A \nabla v)+\left(v-v_{\Gamma}\right)\right)_{\Gamma} \\
= & \mathcal{A}_{1}(u, v)+\left(n \cdot A \nabla u, B^{-1}(n \cdot A \nabla v)\right)_{\Gamma} \\
& -\left(n \cdot A \nabla u, B^{-1}(n \cdot A \nabla v)+\left(v-v_{\Gamma}\right)\right)_{\Gamma} \\
& -\left(B^{-1}(n \cdot A \nabla u)+\left(u-u_{\Gamma}\right), n \cdot A \nabla v\right)_{\Gamma} \\
& +\left(B^{-1}(n \cdot A \nabla u)+\left(u-u_{\Gamma}\right), \tau\left(B^{-1}(n \cdot A \nabla v)+\left(v-v_{\Gamma}\right)\right)_{\Gamma}\right.
\end{aligned}
$$

where the last two terms are zero due to the interface condition and the resulting form on the right hand side is symmetric. Furthermore, $\tau$ is a stabilization parameter (a $2 \times 2$ matrix) of the form

$$
\tau=\sum_{i=1}^{2} \tau_{i} e_{i} \otimes e_{i}, \quad \tau_{i}=\frac{\lambda_{i} \beta}{\lambda_{i} h+\beta} \quad i=1,2
$$

where $\beta$ is a positive parameter and we recall that $\lambda_{i}$ and $e_{i}$ are the eigenvalues and eigenvectors of $B$. The parameter $\beta$ is chosen so that

$$
\|n\|_{A, \infty, \Gamma}^{2}=\sum_{i=1}^{2}\left\|n_{i}\right\|_{A_{i}, \infty, \Gamma}^{2} \lesssim \beta
$$

where $\|w\|_{A_{i}, \infty, \Gamma}=\left\|\left(w \cdot A_{i} \cdot w\right)^{1 / 2}\right\|_{\infty, \Gamma}$. We next show some technical estimates for the stabilization parameter, in particular, we show that $\tau$ is uniformly bounded as a function of $\lambda_{i} \in(0, \infty)$.

Lemma 4 For all eigenvalues $\lambda_{i} \in(0, \infty), i=1,2$, of $B$, the following estimates related to the stabilization parameter $\tau$ hold

$$
\begin{aligned}
& \left\|B^{-1} \tau B^{-1}-B^{-1}\right\|_{L^{\infty}(\Gamma)} \leq \frac{h}{\beta} \\
& \left\|\left(B^{-1} \tau-I\right) \tau^{-1 / 2}\right\|_{L^{\infty}(\Gamma)} \leq\left(\frac{h}{\beta}\right)^{1 / 2}
\end{aligned}
$$




$$
\|\tau\|_{L^{\infty}(\Gamma)} \leq \frac{\beta}{h}
$$

Proof First we recall that for any symmetric matrix $D$ it holds

$$
\|D\|_{\mathbb{R}^{d}} \lesssim \max _{i}\left|\gamma_{i}\right|
$$

where $\gamma_{i}$ are the eigenvalues of $D$. To prove (105) we write $B$ in terms of its eigenvalues $\lambda_{i}$ and eigenvectors $e_{i}$,

$$
B=\sum_{i=1}^{2} \lambda_{i} e_{i} \otimes e_{i}
$$

and using the definition (103) of $\tau$ we obtain the identity

$$
B^{-1} \tau B^{-1}-B^{-1}=\sum_{i=1}^{2}\left(\frac{\tau_{i}}{\lambda_{i}}-1\right) \frac{1}{\lambda_{i}} e_{i} \otimes e_{i}
$$

Here we have the following estimate of the eigenvalues

$$
\left|\left(\frac{\tau_{i}}{\lambda_{i}}-1\right) \frac{1}{\lambda_{i}}\right|=\left|\left(\frac{\beta}{\lambda_{i} h+\beta}-1\right) \frac{1}{\lambda_{i}}\right|=\frac{h}{\lambda_{i} h+\beta} \leq \frac{h}{\beta}
$$

which in view of (108) completes the verification of (105). Next, for (106) we have

$$
\left(B^{-1} \tau-I\right) \tau^{-1 / 2}=\sum_{i=1}^{2}\left(\frac{\tau_{i}}{\lambda_{i}}-1\right) \frac{1}{\tau_{i}^{1 / 2}} e_{i} \otimes e_{i}
$$

and

$$
\begin{aligned}
& \left|\left(\frac{\tau_{i}}{\lambda_{i}}-1\right) \frac{1}{\tau_{i}^{1 / 2}}\right|=\left|\left(\frac{\beta}{\lambda_{i} h+\beta}-1\right)\left(\frac{\lambda_{i} h+\beta}{\lambda_{i} \beta}\right)^{1 / 2}\right| \\
& \quad=\frac{\lambda_{i} h}{\lambda_{i} h+\beta}\left(\frac{\lambda_{i} h+\beta}{\lambda_{i} \beta}\right)^{1 / 2}=\left(\frac{\lambda_{i} h}{\lambda_{i} h+\beta}\right)^{1 / 2}\left(\frac{h}{\beta}\right)^{1 / 2} \leq\left(\frac{h}{\beta}\right)^{1 / 2}
\end{aligned}
$$

which proves (106). The final bound (107) is a direct consequence of the definition of $\tau$ and the estimate

$$
\frac{\lambda_{i} \beta}{\lambda_{i} h+\beta} \leq \frac{\lambda_{i} \beta}{\lambda_{i} h} \leq \frac{\beta}{h}
$$

Remark 8 The choice of $\tau_{i}$ can be further refined as follows

$$
\tau_{i}=\frac{\lambda_{i} \beta_{i}}{\lambda_{i} h+\beta_{i}} \quad i=1,2
$$


with

$$
\sum_{j=1}^{2}\left\|n_{j}\right\|_{A_{j}, \infty, \Gamma}^{2}\left|e_{i j}\right|^{2} \lesssim \beta_{i}
$$

where $e_{i}=\left[\begin{array}{ll}e_{i 1} & e_{i 2}\end{array}\right]^{T}$. This approach is beneficial in situations where the components of $e_{i}$ are very different and there is a large difference between the $\left\|n_{j}\right\|_{A_{j}, \infty, \Gamma}^{2}$ with $j=1$ and $j=2$.

The robust finite element method. Find $u_{h} \in V_{h}$ such that

$$
\mathcal{A}_{h}^{R}\left(u_{h}, v\right)=\mathcal{A}^{R}\left(u_{h}, v\right)+s_{h}\left(u_{h}, v\right)=L(v) \quad \forall v \in V_{h}
$$

where

$$
\begin{aligned}
\mathcal{A}^{R} & (v, w) \\
= & \mathcal{A}_{1}(v, w)+\left(n \cdot A \nabla v, B^{-1}(n \cdot A \nabla w)\right)_{\Gamma} \\
& -\left(n \cdot A \nabla v, B^{-1}(n \cdot A \nabla w)+\left(w-w_{\Gamma}\right)\right)_{\Gamma} \\
& -\left(B^{-1}(n \cdot A \nabla v)+\left(v-v_{\Gamma}\right), n \cdot A \nabla w\right)_{\Gamma} \\
& +\left(B^{-1}(n \cdot A \nabla v)+\left(v-v_{\Gamma}\right), \tau\left(B^{-1}(n \cdot A \nabla w)+\left(w-w_{\Gamma}\right)\right)\right)_{\Gamma}
\end{aligned}
$$

It follows by the design of $\mathcal{A}^{R}$ that for a sufficiently smooth exact solution $u \in \tilde{V}$ of the problem (36) there holds

$$
\mathcal{A}(u, v)=\mathcal{A}^{R}(u, v)=L(v), \quad \forall v \in\left(V \cap H^{2}\left(\Omega_{1} \cup \Omega_{2} \cup \Gamma\right)+V_{h}\right.
$$

As a consequence we immediately get the Galerkin orthogonality

Lemma 5 Let $u \in \tilde{V}$ be the solution of (36) and $u_{h} \in V_{h}$ the solution of (118) then there holds

$$
\mathcal{A}^{R}\left(u-u_{h}, v\right)=s_{h}\left(u_{h}, v\right) \quad \forall v \in V_{h}
$$

Proof The proof follows by combining (124) and (118).

\section{Error estimates}

\subsection{The energy norm}

We introduce the energy norm

$$
\begin{aligned}
\|v\|_{h}^{2}= & \sum_{i=1}^{2}\left\|\nabla v_{i}\right\|_{A_{i}, \Omega_{i}}^{2}+h\left\|\nabla v_{i}\right\|_{A_{i}, \Gamma}^{2}+\|v\|_{S_{h}}^{2} \\
& +\left\|\nabla_{\Gamma} v_{\Gamma}\right\|_{A_{\Gamma}, \Gamma}^{2}+\left\|v-v_{\Gamma}\right\|_{\tau, \Gamma}^{2}
\end{aligned}
$$

where $\|w\|_{\psi, \omega}^{2}=\int_{\omega} \psi w^{2}$ is the $\psi$ weighted $L^{2}$ norm over the set $\omega$. 


\subsection{Interpolation error estimates}

We begin by introducing interpolation operators and derive the basic approximation error estimates. Then collecting the estimates we show an interpolation error estimate in the energy norm (126). The basic idea in the construction of the interpolation operators is to use an extension of the solution outside of the domain and then employ a syandard weak type interpolation operator. The extension operator is stable with respect to Sobolev norms.

The Scott-Zhang interpolant. Given a mesh $\mathcal{T}_{h}$ covering a domain $O_{h}$ and the space of piecewise linear continuous finite elements $W_{h}$, a Scott-Zhang interpolation operator $\pi_{h, S Z}: H^{1}\left(\Omega_{h}\right) \rightarrow W_{h}$ satisfies the element wise estimate

$$
\left\|v-\pi_{h, S Z} v\right\|_{H^{m}(T)} \lesssim h^{2-m}\|v\|_{H^{2}(\mathcal{N}(T))}, \quad m=0,1
$$

where $\mathcal{N}(T)$ is the set of all elements in $\mathcal{T}_{h, i}$ that share a node with $T$. More precisely, we employ a Scott-Zhang interpolation operator that averages on all elements sharing a node for interior nodes, and for nodes at the boundary $\partial \Omega$ the average is taken over all faces on the boundary sharing the node leading to exact preservation of homogeneous boundary conditions. See [33] for further details.

Bulk domain fields. It is shown in [34, Section 2.3, Theorem 5] that there is an extension operator $E_{i}: H^{s}\left(\Omega_{i}\right) \rightarrow H^{s}\left(\mathbb{R}^{d}\right)$, not dependent on $s \geq 0$, which is stable in the sense that

$$
\left\|E_{i} v_{i}\right\|_{H^{s}\left(\mathbb{R}^{d}\right)} \lesssim\left\|v_{i}\right\|_{H^{s}\left(\Omega_{i}\right)}
$$

We define the interpolation operator $\pi_{h, i}: H^{1}\left(\mathcal{N}\left(\mathcal{T}_{h, i}\right)\right) \rightarrow V_{h, i}$ by

$$
\pi_{h, i} v_{i}=\pi_{h, i, S Z} E_{i} v
$$

where $\pi_{h, i, S Z}: H^{1}\left(\mathcal{N}\left(\mathcal{T}_{h, i}\right)\right) \rightarrow V_{h, i}$ is the Scott-Zhang interpolant and $\mathcal{N}\left(\mathcal{T}_{h, i}\right)$ is the set of elements sharing a node with an element in $\mathcal{T}_{h, i}$. We then have the error estimate

$$
\left\|v_{i}-\pi_{h, i} v\right\|_{H^{m}\left(\Omega_{i}\right)} \lesssim h^{2-m}\left\|v_{i}\right\|_{H^{2}\left(\Omega_{i}\right)} \quad m=0,1
$$

Proof Using the notation $\rho_{i}=v_{i}-\pi_{h, i} v_{i}$ we obtain

$$
\left\|\rho_{i}\right\|_{H^{m}\left(\Omega_{i}\right)} \lesssim\left\|\rho_{i}\right\|_{H^{m}\left(\mathcal{T}_{h, i}\right)} \lesssim h^{2-m}\left\|E_{i} u_{i}\right\|_{H^{2}\left(\mathcal{N}\left(\mathcal{T}_{h, i}\right)\right)} \lesssim h^{2-m}\left\|u_{i}\right\|_{H^{2}\left(\Omega_{i}\right)}^{2}
$$

where we used the interpolation error estimate (128) and finally the stability (129) of the extension operator $E_{i}$.

Interface field. Let $p_{\Gamma}: U_{\delta}(\Gamma) \rightarrow \Gamma$ be the closest point mapping from the tubular neighborhood $U_{\delta}(\Gamma)=\{x: \operatorname{dist}(x, \Gamma)<\delta\}$ to $\Gamma$, which is well defined for all $\delta \in\left(0, \delta_{0}\right]$ for some $\delta_{0}>0$. Define the extension operator $E_{\Gamma}: L^{2}(\Gamma) \rightarrow L^{2}\left(U_{\delta}(\Gamma)\right)$ by $E_{\Gamma} v=v \circ p_{\Gamma}$. Since $\Gamma$ is smooth we have the stability estimate

$$
\left\|E_{\Gamma} v_{\Gamma}\right\|_{H^{s}\left(U_{\delta}(\Gamma)\right)} \lesssim \delta^{1 / 2}\left\|v_{\Gamma}\right\|_{H^{s}(\Gamma)}
$$


Observe also that since $n_{\Gamma} \cdot \nabla E_{\Gamma} v_{\Gamma}=0$ by construction, and then assuming $s>3 / 2$ in (133), we see that

$$
s_{h, \Gamma}\left(E_{\Gamma} v_{\Gamma}, w\right)=0, \quad \forall w \in V_{h, \Gamma}+H^{\frac{3}{2}+\epsilon}\left(O_{h, \Gamma}\right)
$$

We define the interpolation operator $\pi_{h, \Gamma}: H^{1}(\Gamma) \rightarrow V_{h, \Gamma}$ by

$$
\pi_{h, \Gamma} v_{\Gamma}=\left.\left(\pi_{h, \Gamma, S Z} E_{\Gamma} v_{\Gamma}\right)\right|_{O_{h, \Gamma}}
$$

Here $\pi_{h, \Gamma, S Z}: H^{1}\left(\mathcal{N}\left(\mathcal{T}_{h, \Gamma}\right)\right) \rightarrow V_{h, \Gamma}$ is the Scott-Zhang interpolation operator defined on the set $\mathcal{N}\left(\mathcal{T}_{h, \Gamma}\right)$ of all elements that share a node with an element in $\mathcal{T}_{h, \Gamma}$. We also note that there is $\delta \sim h$ such that

$$
\mathcal{N}\left(\mathcal{T}_{h, \Gamma}\right) \subset U_{\delta}(\Gamma)
$$

We have the error estimate

$$
\left\|v-\pi_{h, \Gamma} v\right\|_{H^{m}(\Gamma)} \lesssim h^{2-m}\|v\|_{H^{2}(\Gamma)} \quad m=0,1
$$

Proof Using the element wise trace inequality

$$
\|w\|_{\Gamma \cap T}^{2} \lesssim h^{-1}\|w\|_{T}^{2}+h\|\nabla w\|_{T}^{2} \quad w \in H^{1}(T)
$$

see $[23,36]$, we obtain after summation over all elements $T \in \mathcal{T}_{h, \Gamma}$,

$$
\|w\|_{\Gamma}^{2} \lesssim h^{-1}\|w\|_{\mathcal{T}_{h, \Gamma}}^{2}+h\|\nabla w\|_{\mathcal{T}_{h, \Gamma}}^{2} \quad w \in H^{1}\left(\mathcal{T}_{h, \Gamma}\right)
$$

Applying (139) with $w=\nabla_{\Gamma}^{m} \rho$ gives

$$
\begin{aligned}
\left\|\nabla_{\Gamma}^{m} \rho\right\|_{\Gamma}^{2} & \lesssim h^{-1}\left\|\nabla^{m} \rho\right\|_{\mathcal{T}_{h, \Gamma}}^{2}+\delta\left\|\nabla^{m+1} \rho\right\|_{\mathcal{T}_{h, \Gamma}}^{2} \\
& \lesssim\left(h^{-1} h^{2(2-m)}+h h^{2(1-m)}\right)\left\|E_{\Gamma} v\right\|_{H^{2}\left(\mathcal{N}\left(\mathcal{T}_{h, \Gamma}\right)\right)}^{2} \\
& \lesssim\left(h^{-1} h^{2(2-m)}+h h^{2(1-m)}\right)\left\|E_{\Gamma} v\right\|_{H^{2}\left(U_{\delta}(\Gamma)\right)}^{2} \\
& \lesssim \delta\left(h^{-1} h^{2(2-m)}+h h^{2(1-m)}\right)\left\|E_{\Gamma} v\right\|_{\Gamma}^{2} \\
& \lesssim h^{2(2-m)}\|v\|_{H^{2}(\Gamma)}^{2}
\end{aligned}
$$

where we used, the interpolation error estimate (128), the inclusion (136), and the stability (133) of the extension operator $E_{\Gamma}$.

We finally define the interpolation operator $\pi_{h}: V \rightarrow V_{h}$ as follows

$$
\pi_{h} v=\left(\pi_{h, 1} E_{1} v_{1}, \pi_{h, 2} E_{2} v_{2}, \pi_{h, \Gamma} E_{\Gamma} v_{\Gamma}\right)
$$


Lemma 6 There is a constant not dependent on the matrix B, in the interface condition (3), such that

$$
\|\| v-\pi_{h} v \|_{h} \lesssim h\left(\sum_{i=1}^{2}\left\|v_{i}\right\|_{H^{2}\left(\Omega_{i}\right)}+\left\|v_{\Gamma}\right\|_{H^{2}(\Gamma)}\right)
$$

Proof Let $v-\pi_{h} v=\rho$ be the interpolation error. Using the triangle inequality, the estimate $\tau \lesssim h^{-1}$, see (107), and the fact that the coefficients $A_{i}$ are uniformly bounded, we obtain

$$
\begin{aligned}
\|\rho\|_{h}^{2}= & \sum_{i=1}^{2}\left\|\nabla \rho_{i}\right\|_{A_{i}, \Omega_{i}}^{2}+h\left\|\nabla \rho_{i}\right\|_{A_{i}, \Gamma}^{2} \\
& +\left\|\nabla_{\Gamma} \rho_{\Gamma}\right\|_{A_{\Gamma}, \Gamma}^{2}+\left\|\rho_{i}-\rho_{\Gamma}\right\|_{\tau, \Gamma}^{2}+\|\rho\|_{S_{h}}^{2} \\
\lesssim & \sum_{i=1}^{2} \underbrace{\left\|\nabla \rho_{i}\right\|_{\Omega_{i}}^{2}}_{I_{i}}+\underbrace{h\left\|\nabla \rho_{i}\right\|_{\Gamma}^{2}+h^{-1}\left\|\rho_{i}\right\|_{\Gamma}^{2}}_{I I_{i}}+\underbrace{\left\|\rho_{i}\right\|_{S_{h, i}}^{2}}_{I I I_{i}} \\
& +\underbrace{\left\|\nabla_{\Gamma} \rho_{\Gamma}\right\|_{\Gamma}^{2}+h^{-1}\left\|\rho_{\Gamma}\right\|_{\Gamma}^{2}}_{I V}+\underbrace{\left\|\rho_{\Gamma}\right\|_{S_{h, \Gamma}}^{2}}_{V}
\end{aligned}
$$

We proceed with estimates of the terms on the right hand side. Using (131) we directly have

$$
I_{i}=\left\|\nabla \rho_{i}\right\|_{\Omega_{i}}^{2} \lesssim h^{2}\left\|v_{i}\right\|_{H^{2}\left(\Omega_{i}\right)}
$$

Next using the trace inequality

$$
\|w\|_{\Gamma}^{2} \lesssim \delta^{-1}\|w\|_{U_{\delta}(\Gamma) \cap \Omega_{i}}^{2}+\delta\|\nabla w\|_{U_{\delta}(\Gamma) \cap \Omega_{i}}^{2} \quad w \in H^{1}\left(U_{\delta}(\Gamma) \cap \Omega_{i}\right)
$$

with $\delta \sim h$ we get

$$
\begin{aligned}
I I_{i}= & h\left\|\nabla \rho_{i}\right\|_{\Gamma}^{2}+h^{-1}\left\|\rho_{i}\right\|_{\Gamma}^{2} \\
\lesssim & \delta^{-1} h\left\|\nabla \rho_{i}\right\|_{U_{\delta}(\Gamma) \cap \Omega_{i}}^{2}+\delta h\left\|\nabla^{2} \rho_{i}\right\|_{U_{\delta}(\Gamma) \cap \Omega_{i}}^{2} \\
& +\delta^{-1} h^{-1}\left\|\rho_{i}\right\|_{U_{\delta}(\Gamma) \cap \Omega_{i}}^{2}+\delta h^{-1}\left\|\nabla \rho_{i}\right\|_{U_{\delta}(\Gamma) \cap \Omega_{i}}^{2} \\
\lesssim & \delta^{-1} h^{-1}\left\|\rho_{i}\right\|_{\Omega_{i}}^{2}+\left(\delta h^{-1}+\delta^{-1} h\right)\left\|\nabla \rho_{i}\right\|_{\Omega_{i}}^{2}+\delta h\left\|\nabla^{2} \rho_{i}\right\|_{\Omega_{i}}^{2} \\
\lesssim & h^{2}\left\|v_{i}\right\|_{H^{2}\left(\Omega_{i}\right)}^{2}
\end{aligned}
$$

where we at last used (131). For Term IV, applying (137) yields,

$$
I V=h\left\|\nabla \rho_{i}\right\|_{\Gamma}^{2}+h^{-1}\left\|\rho_{i}\right\|_{\Gamma}^{2} \lesssim \sum_{m=0}^{2} h^{2(m-1)}\left\|\nabla^{m} \rho_{i}\right\|_{\Omega_{i}}^{2}
$$


Term $I I I_{i}$ is estimate using the element wise trace inequality

$$
\|w\|_{F}^{2} \lesssim h^{-1}\|w\|_{T}^{2}+h\|\nabla w\|_{T}^{2}
$$

which gives

$$
\begin{aligned}
I I I_{i} & =\left\|\rho_{i}\right\|_{S_{h, i}}^{2} \lesssim \sum_{F \in \mathcal{F}_{h, i}} h\left\|\left[n \cdot \nabla \rho_{i}\right]\right\|_{F}^{2} \\
& \lesssim \sum_{F \in \mathcal{F}_{h, i}}\left\|\nabla \rho_{i}\right\|_{\mathcal{T}_{h, i}(F)}^{2}+h\left\|\nabla^{2} \rho_{i}\right\|_{\mathcal{T}_{h, i}(F)}^{2} \lesssim\|v\|_{H^{m}\left(O_{h, i}\right)}^{2}
\end{aligned}
$$

where $\mathcal{T}_{h, i}(F)$ is the set of two elements that share the face $F$. In a similar way show that

$$
V=\left\|\rho_{\Gamma}\right\|_{S_{h, \Gamma}}^{2} \lesssim\|v\|_{H^{m}\left(O_{h, i}\right)}^{2}
$$

see $[7,28]$ for details.

\subsection{Continuity and coercivity}

We utilize the bounds on the stabilization parameter $\tau$ provided by Lemma 4 to prove continuity and coercivity of the form $A_{h}$.

Lemma 7 There is a constant independent of the eigenvalues of $B$, such that for all $v, w \in \tilde{V}+V_{h}$,

$$
\mathcal{A}_{h}^{R}(v, w) \lesssim\left\|v \left|\left\|_{h}|||w|\right\|_{h}\right.\right.
$$

There is a constant independent of the eigenvalues of $B$, such that for all $v \in V_{h}$,

$$
\|\| \|_{h}^{2} \lesssim \mathcal{A}_{h}^{R}(v, v)
$$

Proof (163). Starting from the definition (119), expanding the terms in $\mathcal{A}^{R}$, and using Cauchy-Schwarz we obtain

$$
\begin{aligned}
\mathcal{A}^{R}(v, w)= & \sum_{i=1}^{2}\left(A_{i} \nabla v_{i}, \nabla w_{i}\right)_{\Omega_{i}}+\left(A_{\Gamma} \nabla_{\Gamma} v_{\Gamma}, \nabla_{\Gamma} w_{\Gamma}\right)_{\Gamma} \\
& +\left((n \cdot A \nabla v),\left(B^{-1} \tau B^{-1}-B^{-1}\right)(n \cdot A \nabla w)\right)_{\Gamma} \\
& +\left((n \cdot A \nabla v),\left(B^{-1} \tau-I\right)\left(w-w_{\Gamma}\right)\right)_{\Gamma} \\
& +\left((n \cdot A \nabla w),\left(B^{-1} \tau-I\right)\left(v-v_{\Gamma}\right)\right)_{\Gamma} \\
& +\left(\left(v-v_{\Gamma}\right), \tau\left(w-w_{\Gamma}\right)\right)_{\Gamma}
\end{aligned}
$$




$$
\begin{aligned}
\leq & \sum_{i=1}^{2}\left\|\nabla v_{i}\right\|_{A_{i}, \Omega_{i}}\left\|\nabla w_{i}\right\|_{A_{i}, \Omega_{i}}+\left\|\nabla_{\Gamma} v_{\Gamma}\right\|_{A_{\Gamma}, \Gamma}\left\|\nabla_{\Gamma} w_{\Gamma}\right\|_{A_{i}, \Gamma} \\
& +\|n \cdot A \nabla v\|_{\Gamma}\left\|B^{-1} \tau B^{-1}-B^{-1}\right\|_{L^{\infty}(\Gamma)}\|n \cdot A \nabla w\|_{\Gamma} \\
& +\|n \cdot A \nabla v\|_{\Gamma}\left\|\left(B^{-1} \tau-I\right) \tau^{-1 / 2}\right\|_{L^{\infty}(\Gamma)}\left\|w-w_{\Gamma}\right\|_{\tau, \Gamma} \\
& +\|n \cdot A \nabla w\|_{\Gamma}\left\|\left(B^{-1} \tau-I\right) \tau^{-1 / 2}\right\|_{L^{\infty}(\Gamma)}\left\|v-v_{\Gamma}\right\|_{\tau, \Gamma} \\
& +\left\|v-v_{\Gamma}\right\|_{\tau, \Gamma}\|w-w\|_{\tau, \Gamma} \\
= & \star
\end{aligned}
$$

Using the estimates (105)-(106) we obtain

$$
\begin{aligned}
\star \leq & \sum_{i=1}^{2}\left\|\nabla v_{i}\right\|_{A_{i}, \Omega_{i}}\left\|\nabla w_{i}\right\|_{A_{i}, \Omega_{i}}+\left\|\nabla_{\Gamma} v_{\Gamma}\right\|_{A_{\Gamma}, \Gamma}\left\|\nabla_{\Gamma} w_{\Gamma}\right\|_{A_{\Gamma}, \Gamma} \\
& +\beta^{-1} h\|n \cdot A \nabla v\|_{\Gamma}\|n \cdot A \nabla w\|_{\Gamma} \\
& +\beta^{-1 / 2} h^{1 / 2}\|n \cdot A \nabla v\|_{\Gamma}\left\|w-w_{\Gamma}\right\|_{\tau, \Gamma} \\
& +\beta^{-1 / 2} h^{1 / 2}\|n \cdot A \nabla w\|_{\Gamma}\left\|v-v_{\Gamma}\right\|_{\tau, \Gamma} \\
& +\left\|v-v_{\Gamma}\right\|_{\tau, \Gamma}\|w-w\|_{\tau, \Gamma} \\
\leq & \sum_{i=1}^{2}\left\|\nabla v_{i}\right\|_{A_{i}, \Omega_{i}}\left\|\nabla w_{i}\right\|_{A_{i}, \Omega_{i}}+\left\|\nabla_{\Gamma} v_{\Gamma}\right\|_{A_{\Gamma}, \Gamma}\left\|\nabla_{\Gamma} w_{\Gamma}\right\|_{A_{\Gamma}, \Gamma} \\
& +\left(\beta^{-1}\|n\|_{A, \infty, \Gamma}^{2} h^{1 / 2}\|\nabla v\|_{A, \Gamma} h^{1 / 2}\|\nabla w\|_{\Gamma}\right. \\
& +\left(\beta^{-1}\|n\|_{A, \infty, \Gamma}^{2}\right)^{1 / 2} h^{1 / 2}\|\nabla v\|_{A, \Gamma}\left\|w-w_{\Gamma}\right\|_{\tau, \Gamma} \\
& +\left(\beta^{-1}\|n\|_{A, \infty, \Gamma}^{2}\right)^{1 / 2} h^{1 / 2}\|\nabla w\|_{A, \Gamma}\left\|v-v_{\Gamma}\right\|_{\tau, \Gamma} \\
& +\left\|v-v_{\Gamma}\right\|_{\tau, \Gamma}\|w-w\|_{\tau, \Gamma} \\
\lesssim & \|v\|\left\|_{h}\right\| w \|_{h}
\end{aligned}
$$

where we used the bound $\beta^{-1}\|n\|_{A, \infty, \Gamma}^{2} \lesssim 1$, see (104). By the Cauchy-Schwarz inequality we have $s_{h}(v, w) \lesssim\|v\|\left\|_{h}\right\|\|w\|_{h}$.

(164). To prove the coercivity we have the identity

$$
\begin{aligned}
\mathcal{A}_{h}^{R}(v, v)= & \sum_{i=1}^{2}\left(A_{i} \nabla v_{i}, \nabla v_{i}\right)_{\Omega_{i}}+\left(A_{\Gamma} \nabla_{\Gamma} v_{\Gamma}, \nabla_{\Gamma} v_{\Gamma}\right)_{\Gamma}+s_{h}(v, v) \\
& +\left((n \cdot A \nabla v),\left(B^{-1} \tau B^{-1}-B^{-1}\right)(n \cdot A \nabla v)\right)_{\Gamma} \\
& +2\left((n \cdot A \nabla v),\left(B^{-1} \tau-I\right)\left(v-v_{\Gamma}\right)\right)_{\Gamma} \\
& +\left(\left(v-v_{\Gamma}\right), \tau\left(v-v_{\Gamma}\right)\right)_{\Gamma} \\
\geq & \sum_{i=1}^{2}\left\|\nabla v_{i}\right\|_{A_{i}, \Omega_{i}}^{2}+\left\|\nabla_{\Gamma} v_{\Gamma}\right\|_{A_{\Gamma}, \Gamma}^{2}+\|v\|_{s_{h}}^{2} \\
& -\beta^{-1}\|n\|_{A, \infty, \Gamma}^{2} h\|\nabla v\|_{A, \Gamma}^{2}
\end{aligned}
$$




$$
\begin{aligned}
& -2\left(\beta^{-1}\|n\|_{A, \infty, \Gamma}^{2}\right)^{1 / 2} h^{1 / 2}\|\nabla v\|_{A, \Gamma}\left\|v-v_{\Gamma}\right\|_{\tau, \Gamma} \\
& +\left\|v-v_{\Gamma}\right\|_{\tau, \Gamma}^{2}
\end{aligned}
$$

We conclude the argument as usual by estimating the negative terms as follows

$$
\begin{aligned}
& \beta^{-1}\|n\|_{A, \infty, \Gamma}^{2} h\|\nabla v\|_{A, \Gamma}^{2}+2\left(\beta^{-1}\|n\|_{A, \infty, \Gamma}^{2}\right)^{1 / 2} h^{1 / 2}\|\nabla v\|_{A, \Gamma}\left\|v-v_{\Gamma}\right\|_{\tau, \Gamma} \\
& \leq 3 \beta^{-1}\|n\|_{A, \infty, \Gamma}^{2} h\|\nabla v\|_{A, \Gamma}^{2}+\frac{1}{2}\left\|v-v_{\Gamma}\right\|_{\tau, \Gamma}^{2} \\
& \leq 3 \beta^{-1}\|n\|_{A, \infty, \Gamma}^{2} C_{I}\left(\sum_{i=1}^{2}\left\|\nabla v_{i}\right\|_{A_{i}, \Omega_{i}}^{2}+\|v\|_{S_{h, i}}^{2}\right)+\frac{1}{2}\left\|v-v_{\Gamma}\right\|_{\tau, \Gamma}^{2} \\
& \leq \frac{1}{2}\left(\sum_{i=1}^{2}\left\|\nabla v_{i}\right\|_{A_{i}, \Omega_{i}}^{2}+\|v\|_{S_{h, i}}^{2}\right)+\frac{1}{2}\left\|v-v_{\Gamma}\right\|_{\tau, \Gamma}^{2}
\end{aligned}
$$

Here we used the inverse estimate

$$
h\left\|\nabla v_{i}\right\|_{A_{i}, \Gamma}^{2} \leq C_{I}\left(\left\|\nabla v_{i}\right\|_{A_{i}, \Omega_{i}}^{2}+\|v\|_{s_{h, i}}^{2}\right)
$$

which follows from the inverse bound

$$
h\left\|\nabla v_{i}\right\|_{A_{i}, \Gamma \cap T}^{2} \lesssim h\left\|\nabla v_{i}\right\|_{\Gamma \cap T}^{2} \lesssim\left\|\nabla v_{i}\right\|_{T}^{2} \lesssim\left\|\nabla v_{i}\right\|_{A_{i}, T}^{2}
$$

together with (90), and finally, we chose $\beta$ large enough to guarantee that

$$
3 \beta^{-1}\|n\|_{A, \infty, \Gamma}^{2} C_{I} \leq \frac{1}{2}
$$

We conclude that

$$
\mathcal{A}_{h}^{R}(v, v) \geq \frac{1}{2}\|\| \|_{h}^{2}
$$

which completes the proof.

\subsection{A priori error estimates}

In this section we prove error estimates for the approximate solution $u_{h}$.

Theorem 1 Let $u \in \tilde{V}$ be the solution of (36) and $u_{h} \in V_{h}$ be the solution of (118). Then there is a constant not dependent on the matrix $B$ in the interface condition (3) such that

$$
\|\| u-u_{h} \|_{h} \lesssim h\left(\sum_{i=1}^{2}\left\|u_{i}\right\|_{H^{2}\left(\Omega_{i}\right)}+\left\|u_{\Gamma}\right\|_{H^{2}(\Gamma)}\right)
$$


Under the assumption that $B$ is a constant positive definite matrix and $A_{i}=a_{i} \mathbb{I}_{[d \times d]}$ for $i=1,2$, and $A_{\Gamma}=a_{i} \mathbb{I}_{[(d-1) \times(d-1)]}$ we also have the estimate

$$
\|\| u-u_{h} \|_{h} \lesssim h\left(\sum_{i=1}^{2}\left\|f_{i}\right\|_{L^{2}\left(\Omega_{i}\right)}+\left\|f_{\Gamma}\right\|_{L^{2}(\Gamma)}\right)
$$

with constant independent of $B$.

Proof First we decompose the error in the approximation error and the discrete error $u-u_{h}=u-\pi_{h} u+\pi_{h} u-u_{h}$ and note that by the triangle inequality

$$
\left\|u-u_{h}\right\|_{h} \lesssim\left\|u-\pi_{h} u\right\|_{h}+\left\|\mid \pi_{h} u-u_{h}\right\|_{h} .
$$

The first term on the right hand side is bounded by (146). For the second term on the right hand side, using coercivity (164), Galerkin orthogonality (125), and continuity (163) we obtain

$$
\begin{aligned}
\left\|\pi_{h} u-u_{h}\right\|_{h}^{2} & \lesssim \mathcal{A}_{h}^{R}\left(\pi_{h} u-u_{h}, \pi_{h} u-u_{h}\right) \\
& =\mathcal{A}^{R}\left(\pi_{h} u-u, \pi_{h} u-u_{h}\right)+s_{h}\left(\pi_{h} u, \pi_{h} u-u_{h}\right) \\
& \lesssim\left\|\pi_{h} u-u\right\|\left\|_{h}\right\| \pi_{h} u-u_{h} \|_{h} .
\end{aligned}
$$

In the last inequality we used that if $u^{e}=\left(E u_{1}, E u_{2}, E_{\Gamma} u_{\Gamma}\right) \in \tilde{V}$ then

$$
s_{h}\left(\pi_{h} u, \pi_{h} u-u_{h}\right)=s_{h}\left(\pi_{h} u-u^{e}, \pi_{h} u-u_{h}\right) \lesssim\left\|\pi_{h} u-u \mid\right\|\left\|_{h}\right\| \pi_{h} u-u_{h} \|_{h} .
$$

Thus

$$
\left\|u-u_{h} \mid\right\|_{h} \lesssim\|\| u-\pi_{h} u \|_{h} \lesssim h\left(\sum_{i=1}^{2}\left\|u_{i}\right\|_{H^{2}\left(\Omega_{i}\right)}+\left\|u_{\Gamma}\right\|_{H^{2}(\Gamma)}\right)
$$

where we used the interpolation error estimate (146). To conclude we apply the regularity estimate (42).

Corollary 1 nder the same assumptions as for Theorem 1 there holds

$$
s_{h}\left(u_{h}, u_{h}\right) \lesssim h\left(\sum_{i=1}^{2}\left\|u_{i}\right\|_{H^{2}\left(\Omega_{i}\right)}+\left\|u_{\Gamma}\right\|_{H^{2}(\Gamma)}\right)
$$

Proof Using the triangle inequality we see that

$$
\left\|u_{h}\right\|_{s_{h}} \leq\left\|\pi_{h} u\right\|_{s_{h}}+\left\|\pi_{h} u-u_{h}\right\|_{s_{h}}
$$


The second term on the right hand side is bounded by the arguments of Theorem 1. For the first term on the right hand side recall that by the consistency properties of $s_{h}$ and the construction of $\pi_{h} u$ there holds

$$
s_{h}\left(\pi_{h} u, \pi_{h} u\right)=s_{h}\left(u^{e}-\pi_{h} u, u^{e}-\pi_{h} u\right) .
$$

We conclude the proof by applying (161) and (162).

The following error estimate in the $L^{2}$-norm also holds.

Theorem 2 Let $u \in \tilde{V}$ be the solution of (36) and $u_{h} \in V_{h}$ be the solution of (118). Assume that $B$ is a constant positive definite matrix and $A_{i}=a_{i} \mathbb{I}_{[d \times d]}$ for $i=1,2$, and $A_{\Gamma}=a_{i} \mathbb{I}_{[(d-1) \times(d-1)]}$. Then there holds

$$
\left\|u_{h}-u\right\|_{\Omega}+\left\|u_{h, \Gamma}-u_{\Gamma}\right\|_{\Gamma} \lesssim h^{2}\left(\sum_{i=1}^{2}\left\|f_{i}\right\|_{L^{2}\left(\Omega_{i}\right)}+\left\|f_{\Gamma}\right\|_{L^{2}(\Gamma)}\right)
$$

Proof For $\psi_{i} \in L^{2}\left(\Omega_{i}\right)$ and $\psi_{\Gamma} \in L^{2}(\Gamma)$, let $\varphi=\left(\varphi_{1}, \varphi_{2}, \varphi_{\Gamma}\right) \in \tilde{V}$ be the weak solution to

$$
\mathcal{A}(v, \varphi)=\sum_{i=1}^{2}\left(\psi_{\Omega_{i}}, v_{i}\right)_{\Omega_{i}}+\left(\psi_{\Gamma}, v_{\Gamma}\right)_{\Gamma} \quad \forall v \in V
$$

Then using the regularity result (42) we have

$$
\left\|\varphi_{1}\right\|_{H^{2}\left(\Omega_{1}\right)}+\left\|\varphi_{2}\right\|_{H^{2}\left(\Omega_{2}\right)}+\left\|\varphi_{\Gamma}\right\|_{H^{2}(\Gamma)} \lesssim\left\|\psi_{\Omega}\right\|_{\Omega}+\left\|\psi_{\Gamma}\right\|_{\Gamma}
$$

with constant independent of $B$. Let $e=\left(u_{1}-u_{1, h}, u_{2}-u_{2, h}, u_{\Gamma}-u_{\Gamma, h}\right)$ be the error and observe that using (124),

$$
\begin{aligned}
& \left(\psi_{\Omega}, u_{1}-u_{1, h}\right)_{\Omega_{1}}+\left(\psi_{\Omega}, u_{2}-u_{2, h}\right)_{\Omega_{2}}+\left(\psi_{\Gamma}, u_{\Gamma}-u_{h, \Gamma}\right)_{\Gamma} \\
& \quad=\mathcal{A}(e, \varphi)=\mathcal{A}^{R}(e, \varphi)
\end{aligned}
$$

Applying now the Galerkin orthogonality (125) we see that

$$
\begin{aligned}
\mathcal{A}^{R}(e, \varphi) & =\mathcal{A}^{R}\left(e, \varphi-\pi_{h} \varphi\right)+\mathcal{A}^{R}\left(e, \pi_{h} \varphi\right) \\
& =\mathcal{A}^{R}\left(e, \varphi-\pi_{h} \varphi\right)+s_{h}\left(u_{h}, \pi_{h} \phi\right) \\
& =\mathcal{A}^{R}\left(e, \varphi-\pi_{h} \varphi\right)+s_{h}\left(e, \phi-\pi_{h} \phi\right) \\
& =\mathcal{A}_{h}^{R}\left(e, \varphi-\pi_{h} \varphi\right)
\end{aligned}
$$

By the continuity (163) we can bound the right hand side,

$$
\mathcal{A}_{h}^{R}\left(e, \varphi-\pi_{h} \varphi\right) \lesssim\|\|\left\|_{h}\right\| \varphi-\pi_{h} \varphi \|_{h}
$$


Fig. 1 Elevation of the computed solution on a particular mesh (for $\alpha=1, \xi=1$ )

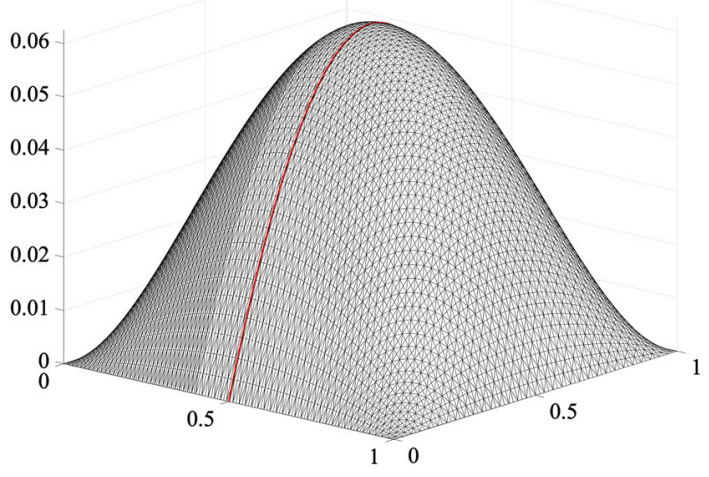

Then applying the approximation (146) and the regularity (216) we have

$$
\left(\psi_{\Omega}, u_{1}-u_{1, h}\right)_{\Omega_{1}}+\left(\psi_{\Omega}, u_{2}-u_{2, h}\right)_{\Omega_{2}}+\left(\psi_{\Gamma}, u_{\Gamma}-u_{h, \Gamma}\right)_{\Gamma} \lesssim h\|e\|_{h}
$$

We conclude by applying Theorem 1 in the right hand side and taking the supremum over the functions $\left(\psi_{1}, \psi_{2}, \psi_{\Gamma}\right)$ such that $\sum_{i=1}^{2}\left\|\psi_{i}\right\|_{\Omega_{i}}^{2}+\left\|\psi_{\Gamma}\right\|_{\Gamma}^{2}=1$.

\section{Numerical examples}

In this section we illustrate the properties of the model and method by presenting some numerical results. In all examples we used $\beta=10$ as a stabilization parameter.

\subsection{Convergence and robustness with respect to conditioning}

We consider a simple example with known exact solution: the domain $(0,1) \times(0,1)$ is cut in half along a vertical line at $x=1 / 2$. We take $A_{1}=A_{2}=A_{\Gamma}=I$ and choose a problem with exact solution $u=x(1-x) y(1-y)$. This solution corresponds (without coupling) to the source terms

$$
f_{i}=2 x(1-x)+2 y(1-y) \quad i=1,2
$$

Since the normal derivative of the exact solution is zero at $x=1 / 2$, it does not contribute to the source term on the interface. We choose $f_{\Gamma}=1 / 2$ corresponding to $u_{\Gamma}=y(1-y) / 4$, and thus $u_{\Gamma}=u$ at $x=1 / 2$. We apply zero Dirichlet boundary conditions on $u$ and on $u_{\Gamma}$ (imposed on the boundary of the band of elements intersected by $(1 / 2, y))$. This is now the solution of (1)-(4) independent of $B$. A sample discrete solution is shown in Fig. 1 with $u_{\Gamma}$ shown as a red line. We did not impose gradient jumps on the band (second term in $s_{h, \Gamma}$ ), normal stabilization proved sufficient in this case. 

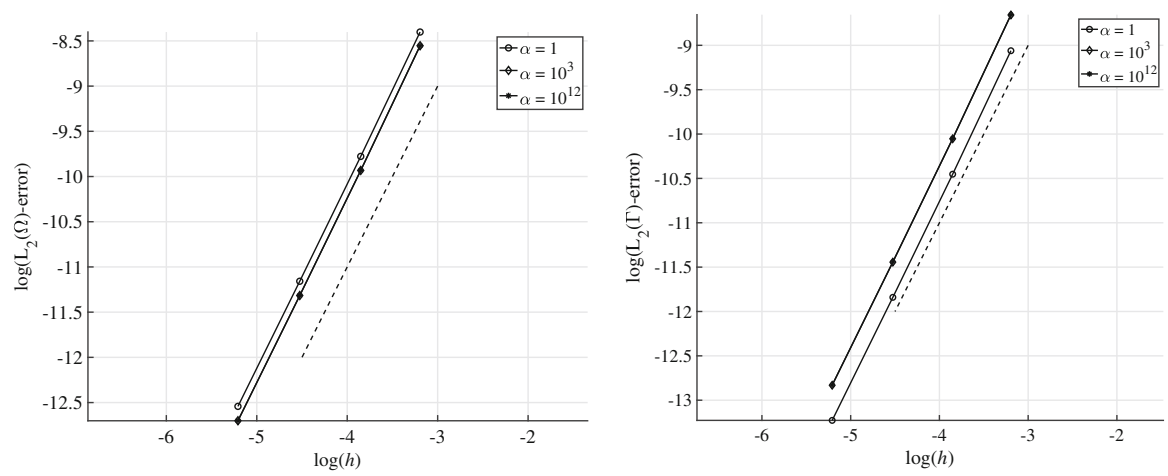

Fig. 2 Convergence in $L_{2}(\Omega)$ and in $L_{2}(\Gamma)$ for varying $\alpha$ with $\xi=1$. Dashed line has inclination 1:2
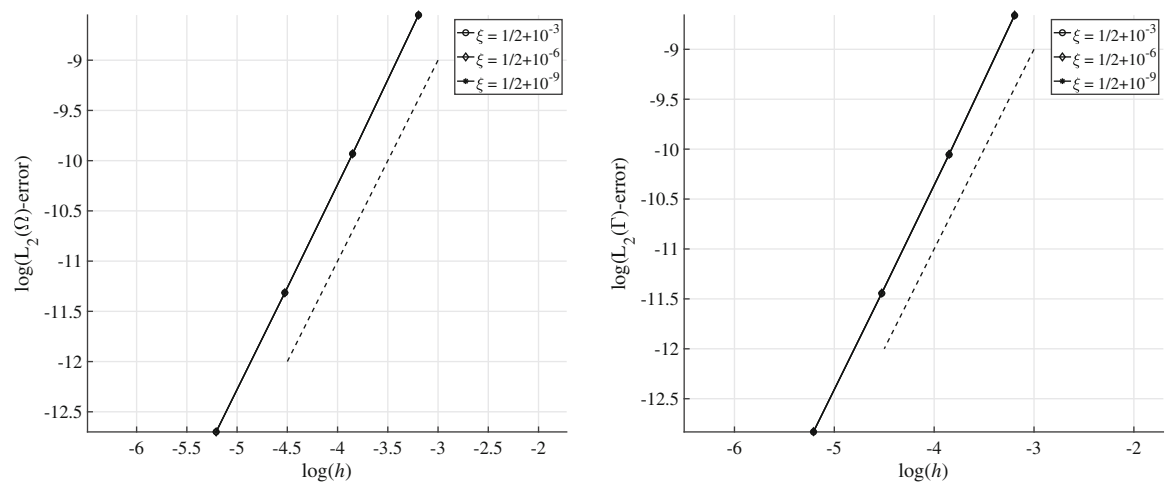

Fig. 3 Convergence in $L_{2}(\Omega)$ and in $L_{2}(\Gamma)$ for varying $\xi$ with $\alpha=1$. Dashed line has inclination 1:2
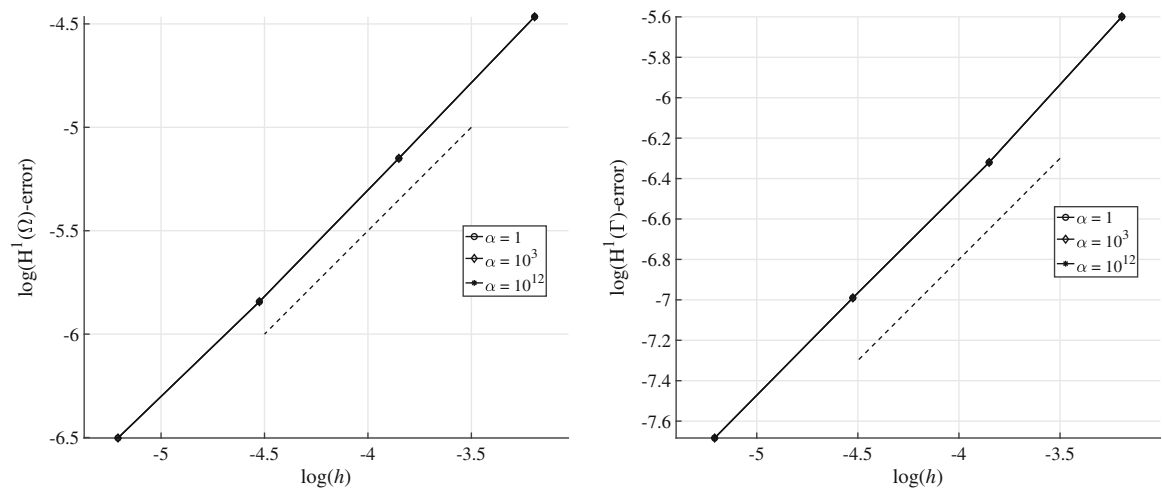

Fig. 4 Convergence in $H^{1}(\Omega)$ and in $H^{1}(\Gamma)$ for varying $\alpha$ with $\xi=1$. Dashed line has inclination 1:1 

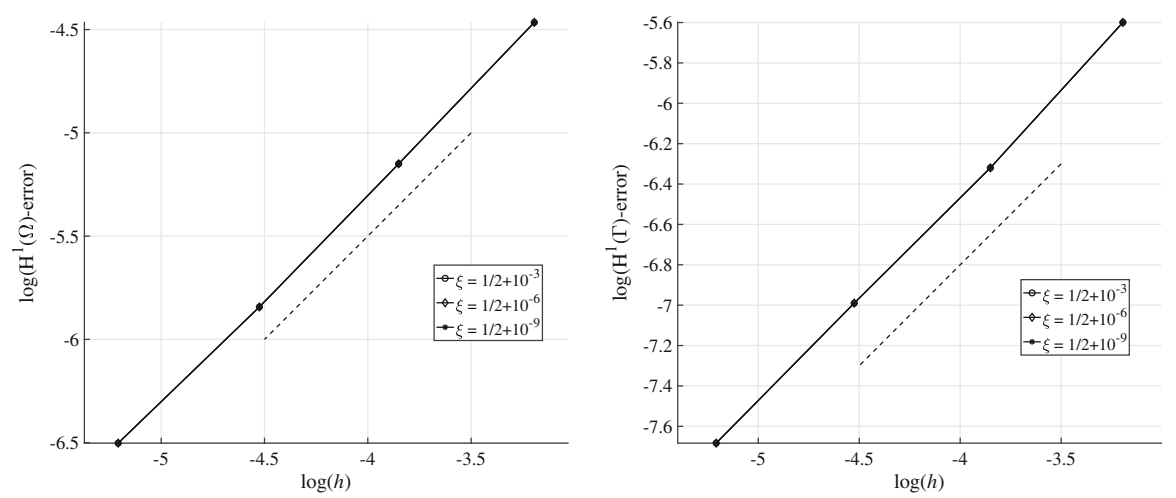

Fig. 5 Convergence in $H^{1}(\Omega)$ and in $H^{1}(\Gamma)$ for varying $\xi$ with $\alpha=1$. Dashed line has inclination 1:1
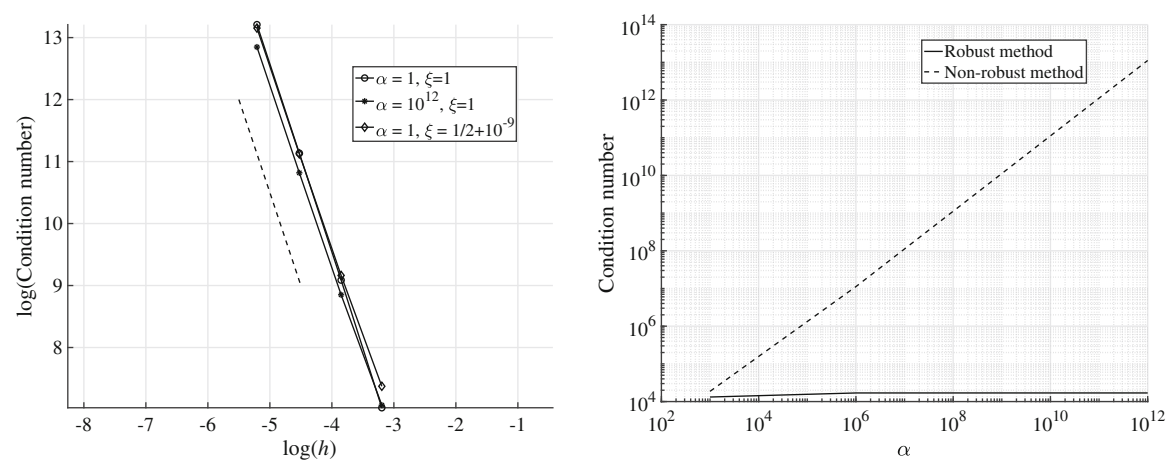

Fig. 6 Left: Condition number as a function of meshsize. Dashed line has inclination 1:2. Right: condition numbers on a fixed mesh with varying $\alpha$ using the robust method (118) and the non-robust method (83)
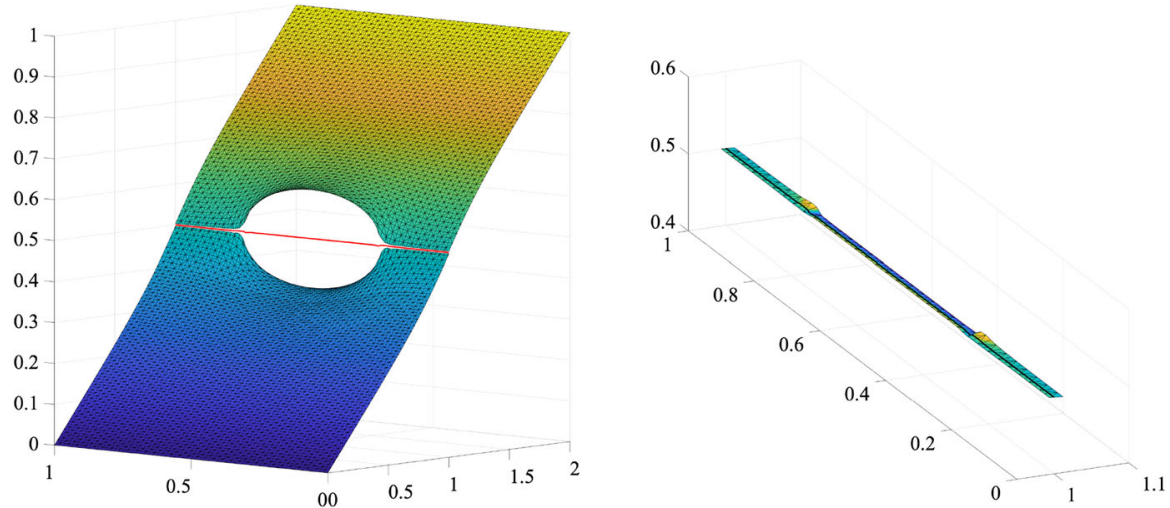

Fig. 7 Elevation of the solution on $\Omega$ and the band containing $\Gamma$ for $\gamma=0$ 

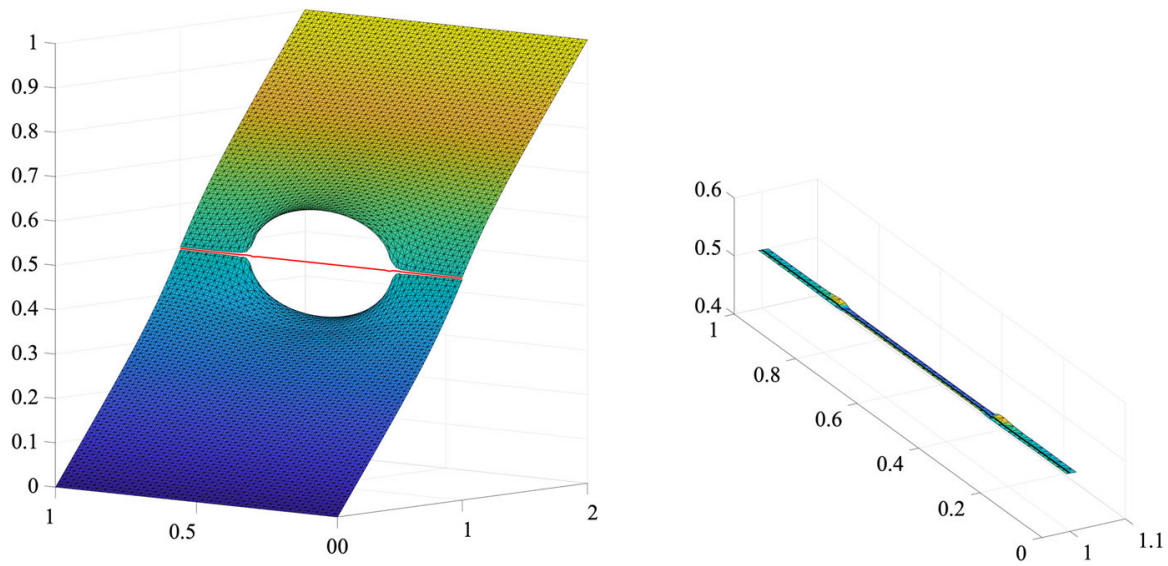

Fig. 8 Elevation of the solution on $\Omega$ and the band containing $\Gamma$ for $\gamma=10^{-2}$
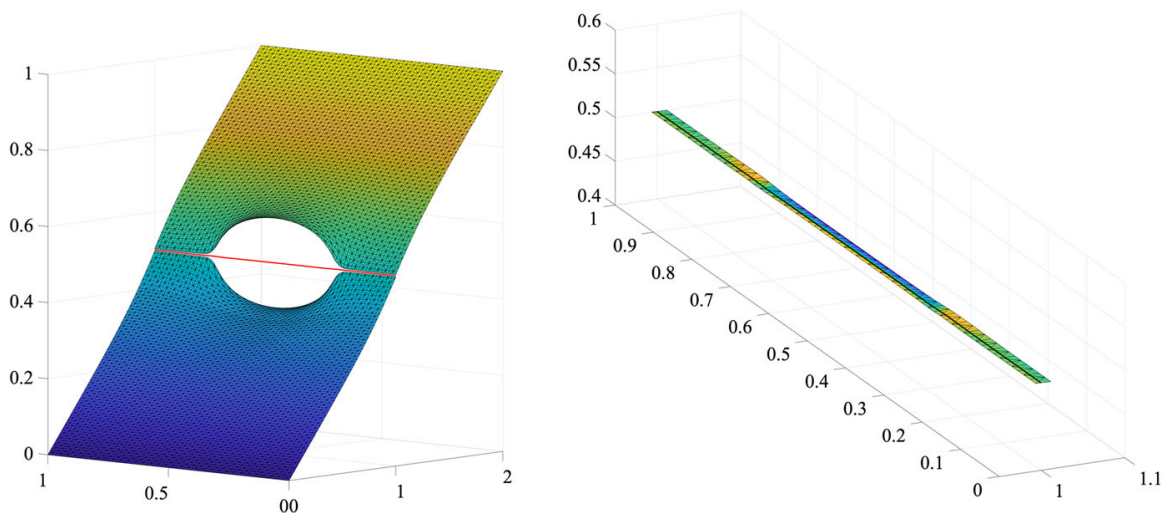

Fig. 9 Elevation of the solution on $\Omega$ and the band containing $\Gamma$ for $\gamma=1$

In Figs. 2, 3, 4 and 5 we show convergence for different choices of parameters in different norms. The method is completely robust with optimal convergence for all choices. In Fig. 6 we show the variation of the condition number (left) with respect to mesh refinement and choice of $\alpha$. The condition number is $O\left(h^{-2}\right)$ as expected and does not grow with $\alpha$. We also show (right) the effect of using the non-robust method (83) which shows a linear dependece on $\alpha$ on a fixed mesh, while no such effect is present in the robust method. This robustness is important since $\alpha$ physically depends on the crack width [29] which is expected to be small.

\subsection{Effect of gradient jump stabilization}

This example is taken from [29] with domain is $(0,2) \times(0,1)$ with Dirichlet data $u=1$ at $x=2$ and $u=0$ at $x=0$. Homogeneous Neumann data were applied at $y=0$ and $y=1$. Data were $f_{i}=f_{\Gamma}=0, A_{1}=A_{2}=I$ and $A_{\Gamma}=a_{\Gamma} d I$ with 
Fig. 10 Computational mesh with interface indicated
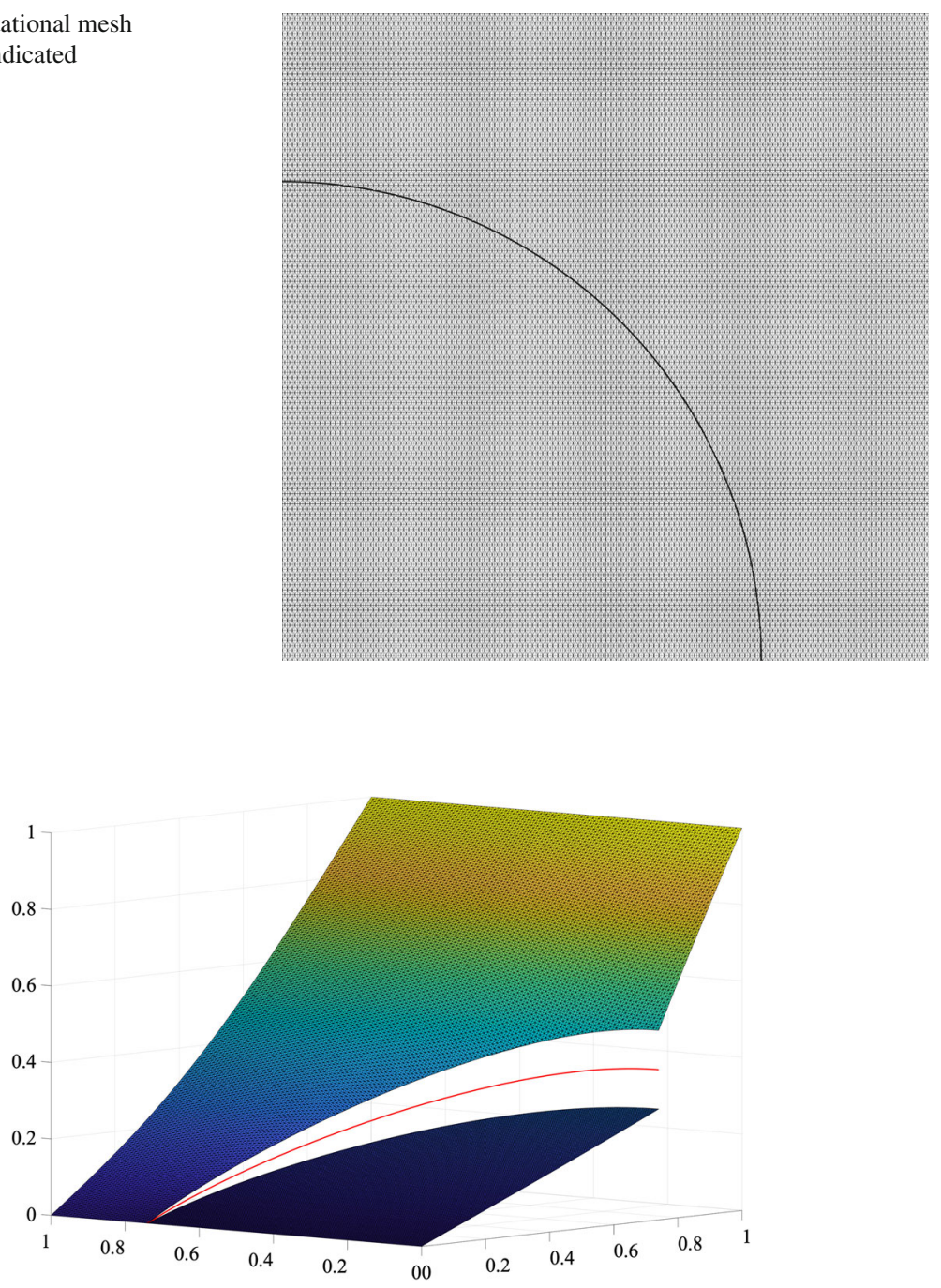

Fig. 11 Elevation for $d=10^{-2}$

$a_{\Gamma}=2 \times 10^{-3}$ for $1 / 4<y<3 / 4, a_{\Gamma}=1$ elsewhere, and with $t=0.01$ (the thickness of the crack). Following [29] we then set $\alpha=2 a_{\Gamma} / d$.

To show the effect of stabilization, we chose to scale $s_{h, i}$ and $s_{h, \Gamma}$ by a parameter $\gamma$. We retained $\beta=10$ and normal stabilization on the band. In Figs. 7, 8 and 9 we show the effect of the parameter $\gamma$. When $\gamma=0$ the jump in diffusion on the interface leads to slight instabilities at $y=1 / 4$ and $y=3 / 4$ which are visible to the eye. These are less pronounced for $\gamma=10^{-2}$ and not significant for $\gamma=1$. The overall solution agrees with that of [29]. 


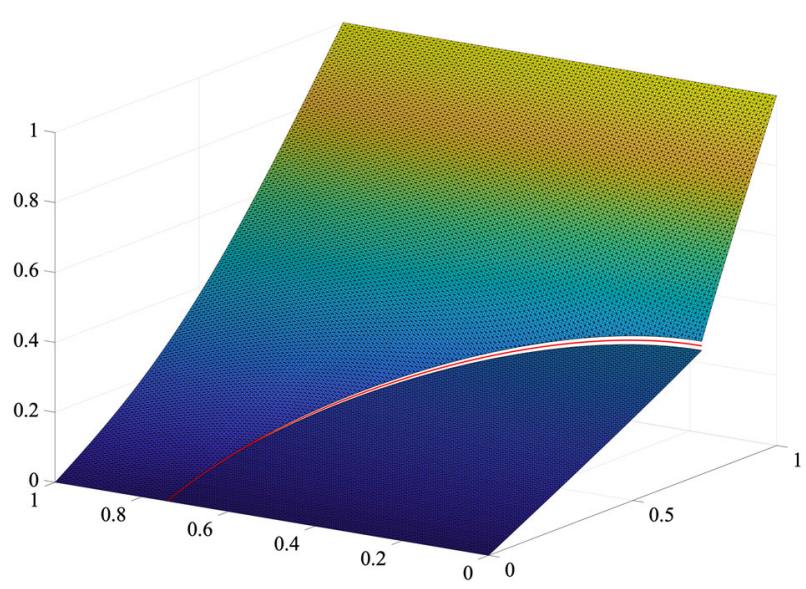

Fig. 12 Elevation for $d=10^{-3}$

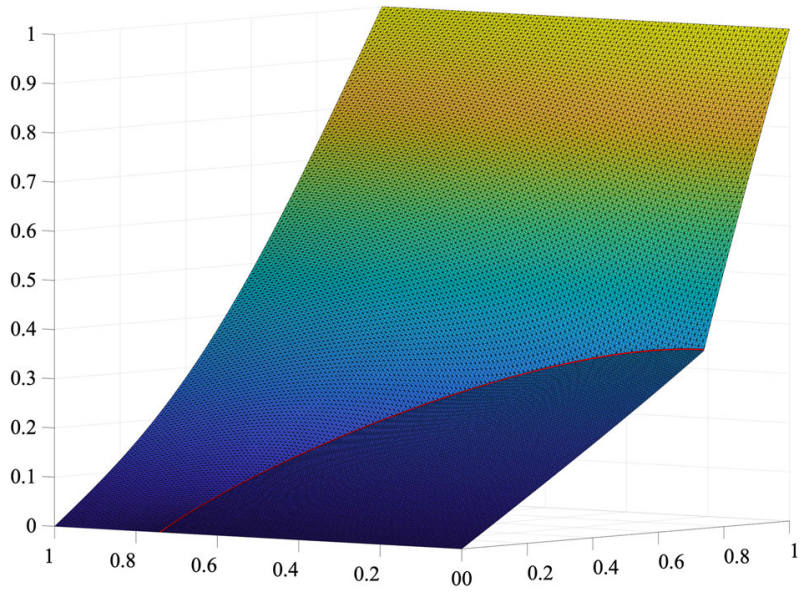

Fig. 13 Elevation for $d=10^{-4}$

\subsection{Physical effect of crack width}

Finally, we show the effect of the crack width with respect to the solution. We used a domain $(0,1) \times(0,1)$ with a quarter circle crack, shown on the computational mesh in Fig. 10. The data were $A_{1}=5 I$ (inside the circle) $A_{2}=I$ (outside the circle) and $a_{\Gamma}=0.1$ with definitions as in Example 5.2. Dirichlet data $u=1$ at $x=1$ and $u=0$ at $x=0$ were used (also on the band) and homogeneous Neumann data on the remaining boundaries. In Figs. 11, 12 and 13 we see the effect of decreasing the interface width by one order of magnitude between figures. The solution rapidly tends to a continuous state. 
Acknowledgements This research was supported in part by EPSRC, UK, Grant No. EP/P01576X/1, the Swedish Foundation for Strategic Research Grant No. AM13-0029, the Swedish Research Council Grant Nos. 2013-4708, 2017-03911, 2018-05262, and Swedish strategic research programme eSSENCE.

Funding Open access funding provided by Jönköping University.

Open Access This article is licensed under a Creative Commons Attribution 4.0 International License, which permits use, sharing, adaptation, distribution and reproduction in any medium or format, as long as you give appropriate credit to the original author(s) and the source, provide a link to the Creative Commons licence, and indicate if changes were made. The images or other third party material in this article are included in the article's Creative Commons licence, unless indicated otherwise in a credit line to the material. If material is not included in the article's Creative Commons licence and your intended use is not permitted by statutory regulation or exceeds the permitted use, you will need to obtain permission directly from the copyright holder. To view a copy of this licence, visit http://creativecommons.org/licenses/by/4.0/.

\section{A Proof of Lemma 2}

We recall that $\omega \subset \mathbb{R}^{d}$ is a domain with $C^{2}$ boundary $\partial \omega$ and we shall show that for $w \in\left[H^{2}(\omega)\right]^{d}$ it holds

$$
\begin{aligned}
(\nabla \cdot w, \nabla \cdot w)_{\omega}= & (w \otimes \nabla, \nabla \otimes w)_{\omega}+2\left(w_{n}, \operatorname{div}_{T} w_{T}\right)_{\partial \omega} \\
& +\left(w_{T}, w_{T}\right)_{\kappa, \partial \omega}+\left(w_{n}, w_{n}\right)_{\operatorname{tr}(\kappa), \partial \omega}
\end{aligned}
$$

Here $w=w_{T}+w_{n} n$ is the decomposition of the vector field $w$ into the tangential and normal components in an open neighborhood $U_{\delta}(\partial \omega)$ consisting of points in $\omega$ with distance less than some $\delta>0$ of the boundary and the normal field on $\partial \omega$ is extended to $U_{\delta}(\partial \omega)$ by composition with the closest point mapping associated with $\partial \omega$. Furthermore, $\kappa=\nabla \otimes n=\nabla^{2} \gamma$ is the tangential curvature tensor of $\partial \omega, \gamma$ is the signed distance function associated with $\partial \omega$ such that $n=\nabla \gamma$. The surface divergence $\operatorname{div}_{T}(w)$ on $\partial \omega$ is defined by $\operatorname{div}_{T}(w)=\operatorname{tr}\left(w \otimes \nabla_{T}\right)$.

Proof Using partial integration we obtain

$$
\begin{aligned}
& (\nabla \cdot w, \nabla \cdot w)_{\omega}=-(w, \nabla(\nabla \cdot v))_{\omega}+\left(w_{n}, \nabla \cdot w\right)_{\partial \omega} \\
& =-(w,(\nabla \otimes w) \cdot \nabla)_{\omega}+\left(w_{n}, \nabla \cdot w\right)_{\partial \omega} \\
& =(w \otimes \nabla, \nabla \otimes w)_{\omega} \underbrace{-(w,(\nabla \otimes w) \cdot n)_{\partial \omega}+\left(w_{n}, \nabla \cdot w\right)_{\partial \omega}}_{\star}
\end{aligned}
$$

where we employed the identity

$$
\nabla(\nabla \cdot w)=(\nabla \otimes w) \cdot \nabla
$$

To evaluate the boundary contribution $\star$ we first note that using the product rule

$$
\nabla w_{n}=\nabla(w \cdot n)=(\nabla \otimes w) \cdot n+(\nabla \otimes n) \cdot w=(\nabla \otimes w) \cdot n+\kappa \cdot w
$$


and thus

$$
(\nabla \otimes w) \cdot n=\nabla w_{n}-\kappa \cdot w
$$

We also note that if $w=w_{T}$ is tangential we have $w_{n}=0$ and

$$
\left(\nabla \otimes w_{T}\right) \cdot n=-\kappa \cdot w_{T}
$$

which also imply

$$
n \cdot\left(\nabla \otimes w_{T}\right) \cdot n=\left(\nabla_{n} w_{T}\right) \cdot n=0
$$

Using (233) the boundary contribution takes the form

$$
\begin{aligned}
\star & =-(w,(\nabla \otimes w) \cdot n)_{\partial \omega}+\left(w_{n}, \nabla \cdot w\right)_{\partial \omega} \\
& =(w, \kappa \cdot w)_{\partial \omega} \underbrace{-\left(w, \nabla w_{n}\right)_{\partial \omega}+\left(w_{n}, \nabla \cdot w\right)_{\partial \omega}}_{\star \star}
\end{aligned}
$$

Continuing $\star \star$ and writing $w=w_{T}+w_{n} n$ we get

$$
\begin{aligned}
\star \star & -\left(w, \nabla w_{n}\right)_{\partial \omega}+\left(w_{n}, \nabla \cdot w\right)_{\partial \omega} \\
= & -\left(w_{n} n, \nabla w_{n}\right)_{\partial \omega}+\left(w_{n}, \nabla \cdot\left(w_{n} n\right)\right)_{\partial \omega} \\
& -\left(w_{T}, \nabla w_{n}\right)_{\partial \omega}+\left(w_{n}, \nabla \cdot w_{T}\right)_{\partial \omega} \\
= & -\left(w_{n} n, \nabla w_{n}\right)_{\partial \omega}+\left(w_{n},\left(\nabla \cdot w_{n}\right) n+w_{n} \nabla \cdot n\right)_{\partial \omega} \\
& -\left(w_{T}, \nabla w_{n}\right)_{\partial \omega}+\left(w_{n}, \nabla \cdot w_{T}\right)_{\partial \omega} \\
= & \left(w_{n}, \operatorname{tr}(\kappa) w_{n}\right)_{\partial \omega} \underbrace{-\left(w_{T}, \nabla w_{n}\right)_{\partial \omega}+\left(w_{n}, \nabla \cdot w_{T}\right)_{\partial \omega}}_{\star \star \star}
\end{aligned}
$$

where we used the product rule $\nabla \cdot\left(w_{n} n\right)=\left(\nabla w_{n}\right) n+w_{n} \nabla \cdot n$, and the identity $\nabla \cdot n=\operatorname{tr}(n \otimes \nabla)=\operatorname{tr}(\kappa)$. Finally, for the last term $\star \star \star$, we split the gradient $\nabla w$ in its tangential and normal components $\nabla w=\nabla_{T} w+n \nabla_{n} w$ and starting from the definition of the divergence as the trace of the derivative of $w$ we get

$$
\begin{aligned}
\nabla \cdot w_{T} & =\operatorname{tr}\left(w_{T} \otimes \nabla\right) \\
& =\operatorname{tr}\left(w_{T} \otimes \nabla_{T}\right)+\operatorname{tr}\left(w_{T} \otimes n \nabla_{n}\right) \\
& =\operatorname{div}_{T} w_{T}+\underbrace{n \cdot\left(w_{T} \otimes \nabla\right) \cdot n}_{=0} \\
& =\operatorname{div}_{T} w_{T}
\end{aligned}
$$

where $\operatorname{div}_{T}$ is the tangential divergence and we used (235). Finally, using Green's formula

$$
-\left(\operatorname{div}_{T} w_{T}, v\right)_{\partial \omega}=\left(w_{T}, \nabla_{T} v\right)_{\partial \omega}
$$


on the closed boundary $\partial \omega$ we obtain

$$
\begin{aligned}
\star \star \star & =-\left(w_{T}, \nabla_{T} w_{n}\right)_{\partial \omega}+\left(w_{n}, \nabla \cdot w_{T}\right)_{\partial \omega} \\
& =2\left(\operatorname{div}_{T}\left(w_{T}\right), w_{n}\right)_{\partial \omega}
\end{aligned}
$$

which concludes the proof.

\section{B Proof of A trace inequality}

We shall prove the trace inequality

$$
\left\|\nabla_{n} v\right\|_{H^{1 / 2}(\Gamma)} \lesssim\|\nabla v\|_{H^{1}\left(\Omega_{i}\right)}
$$

We begin by introducing some notation. Let $\gamma$ be the signed distance function associated with $\Gamma$, let $U_{\delta}(\Gamma)$ be the tubular neighborhood consisting of points $x$ with distance $|\gamma(x)|$ less than $\delta$ to $\Gamma$, let $p$ be the closest point mapping $U_{\delta}(\Gamma) \rightarrow \Gamma$, and recall that since $\Gamma$ is smooth there is $\delta_{0}>0$ such that $p$ is a well defined function on $U_{\delta_{0}}(\Gamma)$. Let $\psi_{\delta}: \Omega_{i} \rightarrow[0,1]$ be a non negative smooth cut off function with support in $U_{\delta}(\Gamma) \cap \Omega_{i}$, such that $\psi=1$ on $\Gamma$ and $\|\psi\|_{L^{\infty}\left(\Omega_{i}\right)} \leq C \delta^{-1}$. Let $n^{e}=n \circ p$ be the extension of $n$ to $U_{\delta_{0}}(\Gamma)$ and using the definition $\|v\|_{H^{1 / 2}(\Gamma)}=\inf _{w \in H^{1}\left(\Omega_{i}\right), w=v \text { on } \Gamma}\|w\|_{H^{1}\left(\Omega_{i}\right)}$ of the $H^{1 / 2}(\Gamma)$ norm we conclude that taking $w=\psi_{\delta} n^{e} \cdot \nabla u_{i}$ we have the inequality

$$
\left\|n \cdot \nabla u_{i}\right\|_{H^{1} / 2(\Gamma)}^{2} \leq\left\|\psi_{\delta} n^{e} \cdot \nabla u_{i}\right\|_{\Omega_{i}}^{2}+\left\|\nabla\left(\psi_{\delta} n^{e} \cdot \nabla u_{i}\right)\right\|_{\Omega_{i}}^{2}
$$

Since $\left\|\psi_{\delta}\right\|_{L^{\infty}\left(\Omega_{i}\right)} \leq 1$ and $\left\|n^{e}\right\|_{L^{\infty}\left(U_{\delta_{0}}(\Gamma)\right) \cap \Omega_{i}}=1$ we have

$$
\left\|\psi_{\delta} n^{e} \cdot \nabla u_{i}\right\|_{\Omega_{i}}^{2} \leq\left\|\nabla u_{i}\right\|_{\Omega_{i}}^{2}
$$

and using the product rule followed by the triangle inequality and standard estimates

$$
\begin{aligned}
\left\|\nabla\left(\psi_{\delta} n^{e} \cdot \nabla u_{i}\right)\right\|_{\Omega_{i}}^{2} \leq & \left\|\nabla \psi_{\delta}\right\|_{L^{\infty}\left(U_{\delta}(\Gamma) \cap \Omega_{i}\right)}\left\|\nabla u_{i}\right\|_{\Omega_{i}}^{2} \\
& +\left\|\nabla n^{e}\right\|_{L^{\infty}\left(U_{\delta}(\Gamma) \cap \Omega_{i}\right)}\left\|\nabla u_{i}\right\|_{\Omega_{i}}^{2}+\left\|\nabla^{2} u_{i}\right\|_{\Omega_{i}}^{2} \\
\leq & C^{2} \delta^{-2}\left\|\nabla u_{i}\right\|_{\Omega_{i}}^{2}+C_{\kappa}^{2}\left\|\nabla u_{i}\right\|_{\Omega_{i}}^{2}+\left\|\nabla^{2} u_{i}\right\|_{\Omega_{i}}^{2} \\
\lesssim & \left\|\nabla u_{i}\right\|_{H^{1}\left(\Omega_{i}\right)}^{2}
\end{aligned}
$$

where $C$ denotes a generic constant and we used the properties of the cut off function. Finally, to compute $D n^{e}$ we recall that the closest point mapping takes the form $p(x)=x-\gamma(x) n^{e}(x)$, and using the chain rule together with the fact that $\nabla \gamma=n^{e}$ we get

$$
D n^{e}=D n D p=D n\left(I-n^{e} \otimes n^{e}-\gamma D n^{e}\right)
$$

Rearranging terms and using the fact that $D n=\kappa$ we get

$$
(I+\gamma \kappa) D n^{e}=\kappa\left(I-n^{e} \otimes n^{e}\right)=\kappa
$$


since $\kappa$ is tangential and therefore

$$
D n^{e}=(I+\gamma \kappa)^{-1} \kappa
$$

For $\gamma \leq \delta$, with $\delta$ small enough we have $\left\|D n^{e}(x)\right\| \lesssim \| \kappa(p(x) \| \lesssim 1$, which completes the proof.

\section{Variable interface coefficient in the regularity estimate}

We show that the regularity estimate (42) also holds for a variable coeffcient $B$ in the interface condition such that $B \in\left[W_{\infty}^{1}(\Gamma)\right]^{2 \times 2}$. We replace identities (54)-(56) with the following estimate

$$
\begin{aligned}
& \left(B\left[u-u_{\Gamma}\right], \Delta_{\Gamma}\left[u-u_{\Gamma}\right]\right)_{\Gamma}=\left(\left(B\left[u-u_{\Gamma}\right]\right) \otimes \nabla_{\Gamma},\left[u-u_{\Gamma}\right] \otimes \nabla_{\Gamma}\right)_{\Gamma}(261) \\
& =\left(B\left(\left[u-u_{\Gamma}\right] \otimes \nabla_{\Gamma}\right),\left[u-u_{\Gamma}\right] \otimes \nabla_{\Gamma}\right)_{\Gamma} \\
& \quad+\left(\left(\nabla_{\Gamma} \otimes B\right)\left[u-u_{\Gamma}\right], \nabla_{\Gamma} \otimes\left[u-u_{\Gamma}\right]\right)_{\Gamma} \\
& \geq\left\|\left[u-u_{\Gamma}\right] \otimes \nabla_{\Gamma}\right\|_{B, \Gamma}^{2} \\
& \quad-\left\|\left(\nabla_{\Gamma} \otimes B\right) B^{-1}\right\|_{L^{\infty}(\Gamma)}\left\|\left[u-u_{\Gamma}\right]\right\|_{B, \Gamma}\left\|\left[u-u_{\Gamma}\right] \otimes \nabla_{\Gamma}\right\|_{B, \Gamma} \\
& \geq \frac{1}{2}\left\|\left[u-u_{\Gamma}\right] \otimes \nabla_{\Gamma}\right\|_{B, \Gamma}^{2}-\frac{1}{2}\left\|\left(\nabla_{\Gamma} \otimes B\right) B^{-1}\right\|_{L^{\infty}(\Gamma)}^{2}\left\|\left[u-u_{\Gamma}\right]\right\|_{B, \Gamma}
\end{aligned}
$$

Here we note that the negative term on the right hand side can be estimated using the energy norm and using the fact that $B$ is positive definite (6) we also have $\|\left(\nabla_{\Gamma} \otimes\right.$ B) $B^{-1}\left\|_{L^{\infty}(\Gamma)} \lesssim c_{\lambda}^{-1}\right\|\left(\nabla_{\Gamma} \otimes B\right) \|_{L^{\infty}(\Gamma)}$.

\section{References}

1. Angot, P., Boyer, F., Hubert, F.: Asymptotic and numerical modelling of flows in fractured porous media. M2AN Math. Model. Numer. Anal. 43(2), 239-275 (2009)

2. Antonietti, P.F., Facciolà, C., Russo, A., Verani, M.: Discontinuous Galerkin approximation of flows in fractured porous media on polytopic grids. SIAM J. Sci. Comput. 41(1), A109-A138 (2019)

3. Boon, W.M., Nordbotten, J.M., Yotov, I.: Robust discretization of flow in fractured porous media. SIAM J. Numer. Anal. 56(4), 2203-2233 (2018)

4. Burman, E., Claus, S., Hansbo, P., Larson, M.G., Massing, A.: CutFEM: discretizing geometry and partial differential equations. Int. J. Numer. Methods Eng. 104(7), 472-501 (2015)

5. Burman, E., Elfverson, D., Hansbo, P., Larson, M.G., Larsson, K.: Hybridized CutFEM for elliptic interface problems. SIAM J. Sci. Comput. 41(5), A3354-A3380 (2019)

6. Burman, E., Hansbo, P.: Fictitious domain finite element methods using cut elements: II. A stabilized Nitsche method. Appl. Numer. Math. 62(4), 328-341 (2012)

7. Burman, E., Hansbo, P., Larson, M.G.: A stabilized cut finite element method for partial differential equations on surfaces: the Laplace-Beltrami operator. Comput. Methods Appl. Mech. Eng. 285, 188207 (2015)

8. Burman, E., Hansbo, P., Larson, M.G.: A simple finite element method for elliptic bulk problems with embedded surfaces. Comput. Geosci. 23(1), 189-199 (2019)

9. Burman, E., Hansbo, P., Larson, M.G., Larsson, K.: Cut finite elements for convection in fractured domains. Comput. Fluids 179, 726-734 (2019) 
10. Burman, E., Hansbo, P., Larson, M.G., Larsson, K., Massing, A.: Finite element approximation of the Laplace-Beltrami operator on a surface with boundary. Numer. Math. 141(1), 141-172 (2019)

11. Burman, E., Hansbo, P., Larson, M.G., Samvin, D.: A cut finite element method for elliptic bulk problems with embedded surfaces. GEM Int. J. Geomath. 10(1), Art. 10, 22 (2019)

12. Burman, E., Hansbo, P., Larson, M.G., Zahedi, S.: Cut finite element methods for coupled bulk-surface problems. Numer. Math. 133(2), 203-231 (2016)

13. Capatina, D., Luce, R., El-Otmany, H., Barrau, N.: Nitsche's extended finite element method for a fracture model in porous media. Appl. Anal. 95(10), 2224-2242 (2016)

14. Chave, F., Di Pietro, D.A., Formaggia, L.: A hybrid high-order method for Darcy flows in fractured porous media. SIAM J. Sci. Comput. 40(2), A1063-A1094 (2018)

15. Chernyshenko, A.Y., Olshanskii, M.A.: An unfitted finite element method for the Darcy problem in a fracture network. J. Comput. Appl. Math. 366, 112424, 14 (2020)

16. Costabel, M., Dauge, M.: Crack singularities for general elliptic systems. Math. Nachr. 235, 29-49 (2002)

17. Costabel, M., Dauge, M., Duduchava, R.: Asymptotics without logarithmic terms for crack problems. Comm. Partial Differ. Equ. 28(5-6), 869-926 (2003)

18. Fumagalli, A., Keilegavlen, E.: Dual virtual element method for discrete fractures networks. SIAM J. Sci. Comput. 40(1), B228-B258 (2018)

19. Grisvard, P.: Elliptic problems in nonsmooth domains, volume 69 of Classics in Applied Mathematics. Society for Industrial and Applied Mathematics (SIAM), Philadelphia, PA: Reprint of the 1985 original [MR0775683]. With a foreword by Susanne C, Brenner (2011)

20. Gross, S., Olshanskii, M.A., Reusken, A.: A trace finite element method for a class of coupled bulkinterface transport problems. ESAIM Math. Model. Numer. Anal. 49(5), 1303-1330 (2015)

21. Hansbo, A., Hansbo, P.: An unfitted finite element method, based on Nitsche's method, for elliptic interface problems. Comput. Methods Appl. Mech. Eng. 191(47-48), 5537-5552 (2002)

22. Hansbo, A., Hansbo, P.: A finite element method for the simulation of strong and weak discontinuities in solid mechanics. Comput. Methods Appl. Mech. Eng. 193(33-35), 3523-3540 (2004)

23. Hansbo, A., Hansbo, P., Larson, M.G.: A finite element method on composite grids based on Nitsche's method. ESAIM: Math. Model. Numer. Anal. 37(3), 495-514 (2003)

24. Hansbo, P., Jonsson, T., Larson, M.G., Larsson, K.: A Nitsche method for elliptic problems on composite surfaces. Comput. Methods Appl. Mech. Eng. 326, 505-525 (2017)

25. Hung, P.H., Sánchez-Palencia, E.: Phénomènes de transmission à travers des couches minces de conductivité élevée. J. Math. Anal. Appl. 47, 284-309 (1974)

26. Juntunen, M., Stenberg, R.: Nitsche's method for general boundary conditions. Math. Comp. 78(267), 1353-1374 (2009)

27. Köppel, M., Martin, V., Roberts, J.E.: A stabilized Lagrange multiplier finite-element method for flow in porous media with fractures. GEM Int. J. Geomath. 10(1), 7 (2019)

28. Larson, M.G., Zahedi, S.: Stabilization of high order cut finite element methods on surfaces. IMA J. Numer. Anal. 40(3), 1702-1745 (2020)

29. Martin, V., Jaffré, J., Roberts, J.E.: Modeling fractures and barriers as interfaces for flow in porous media. SIAM J. Sci. Comput. 26(5), 1667-1691 (2005)

30. Morales, F., Showalter, R.E.: The narrow fracture approximation by channeled flow. J. Math. Anal. Appl. 365(1), 320-331 (2010)

31. Odsæ ter, L. H., Kvamsdal, T., Larson, M.G.: A simple embedded discrete fracture-matrix model for a coupled flow and transport problem in porous media. Comput. Methods Appl. Mech. Eng. 343, 572-601 (2019)

32. Olshanskii, M.A., Reusken, A.: Trace finite element methods for PDEs on surfaces. In: Geometrically Unfitted Finite Element Methods and Applications, volume 121 of Lecture Notes Computer Science Engineering, pp. 211-258. Springer, Cham (2017)

33. Scott, L.R., Zhang, S.: Finite element interpolation of nonsmooth functions satisfying boundary conditions. Math. Comp. 54(190), 483-493 (1990)

34. Stein, E.M.: Singular integrals and differentiability properties of functions. Princeton Mathematical Series, No. 30. Princeton University Press, Princeton (1970)

35. Taylor, M.E.: Partial Differential Equations I. Basic Theory, volume 115 of Applied Mathematical Sciences, 2nd edn. Springer, New York (2011)

36. Wu, H., Xiao, Y.: An unfitted $h p$-interface penalty finite element method for elliptic interface problems. J. Comput. Math. 37(3), 316-339 (2019) 
37. Yedeg, E.L., Wadbro, E., Hansbo, P., Larson, M.G., Berggren, M.: A Nitsche-type method for Helmholtz equation with an embedded acoustically permeable interface. Comput. Methods Appl. Mech. Eng. 304, 479-500 (2016)

Publisher's Note Springer Nature remains neutral with regard to jurisdictional claims in published maps and institutional affiliations. 